\title{
Extensions of the SAFT model for complex association in the bulk and
}

\section{interface}

\author{
Wael A. Fouad, Amin Haghmoradi, Le Wang, Artee Bansal, Ali Al Hammadi, Dilip Asthagiri, \\ Essmaiil Djamali, Kenneth R. Cox, and Walter G. Chapman*
}

Department of Chemical and Biomolecular Engineering, Rice University, Texas 77005, USA

\begin{abstract}
In honor of the 25th anniversary of the SAFT equation of state, this paper presents a brief review of successes in modeling fluid mixtures with associating, polyatomic, and polar components using the SAFT equation of state. Challenges for the association term to predict monomer and dimer fractions in comparison with spectroscopic data are described. To address these challenges and challenges in patchy colloids, extensions to the associating term are reviewed that incorporate multiple bonding at association sites and cooperative association. Approximations in these extensions are validated through comparisons with molecular simulation results. Further, application of the density functional form of the theory to form micelles is briefly reviewed.
\end{abstract}

*Electronic mail: wgchap@ rice.edu, Tel.: 1-713-348-4900, Fax: 1-713-348-5478. 


\section{Introduction}

Complex fluid mixtures, whose thermodynamic properties depend on multiple length scales due to differences in size, shape, or strong directional interactions, have long been challenges for conventional engineering correlations. The SAFT equation of state [1-3] has become widely applied in industry and academia because it provides a tool to predict properties, phase behavior, and microstructure of such complex fluids. Examples of these are mixtures containing hydrogen bonding components and molecules of different sizes and shapes such as polymers and solvents. Further, because it was derived from statistical mechanics, SAFT uses physical model parameters that enable the equation of state to predict fluid properties outside the range of fitted parameters with some confidence. This strong basis in theory also provides the opportunity to extend the SAFT model as additional physics emerges as essential in describing the system. In the following work, we describe some applications of the SAFT model and briefly review recent extensions of the model to incorporate additional physics of association. These are based on a presentation and a series of posters given at the SAFT 2015 conference.

SAFT is based on extensions and simplifications of Wertheim's thermodynamic perturbation theory [4-7] for associating molecules. Similar to a group contribution theory, in the SAFT approach, the fluid of interest is initially considered to be a mixture of unconnected groups or segments. SAFT predicts the free energy change due to chain connectivity (bonding of various segments) to form polyatomic molecules. SAFT also includes an explicit hydrogen bonding contribution to the free energy. In this way, SAFT models molecules as chains of spherical segments that are bonded together and these chain-like molecules can further associate with each other. 
Having SAFT derived from statistical mechanics based theory offers several advantages. The model parameters are physical intermolecular potential parameters similar to those used in molecular simulation. This allows direct comparison with molecular simulation results. In this way, the range of applicability of the chain and association terms have been validated through comparisons with fluid properties calculated from computer simulations of realistic mixtures. If any weak approximations in SAFT or additional physics are identified, the equation of state can be systematically improved by extending the theory. The physical SAFT parameters have been found to behave systematically within a homologous series, allowing prediction from previously modeled systems. This physical basis also provides confidence that the theory can predict results at conditions beyond where the model parameters were fit. Many forms of the SAFT equation of state have been proposed that primarily differ in the model for the segment contribution to the free energy [8-21]. All versions produce similar results in comparison to the experiment as they all use essentially the same association and chain terms derived in the original SAFT equation of state $[1-3,22]$.

The impact of the SAFT equation of state is seen in the large number of research groups and papers from around the world applying SAFT to complex fluid thermodynamics spread over several fields of chemicals, performance polymers, petroleum systems, and biochemical systems. Although SAFT began as a theory for small hydrogen bonding molecules, the equation of state found wide application in modeling properties and phase behavior in systems where molecules show large size asymmetry. The SAFT model has found widest applications as a primary equation of state in designing processes for performance polymers and in modeling asphaltene precipitation in crude oil systems. Applications of the SAFT equation of state are also found in natural gas processing including gas dehydration and $\mathrm{CO}_{2}$ capture; in flow assurance related to 
wax, asphaltene, and gas hydrate phase behavior; in separations related to biofuels and biochemical; in the chemical industry related to mixtures containing alcohols, surfactants, and ionic liquids; and even in predicting fluid phase behavior of nitrogen compounds in the methane lakes on Titan [23]. In supplemental material, we include tables categorizing some applications of various SAFT models from 477 publications. Beyond bulk fluids, density functional theories based on the SAFT framework are applied to nano-structured fluids in materials and biology [24, 25] to provide interfacial properties and predict molecular scale and meso-scale structure. Beyond chemical systems, SAFT has become a primary model in the condensed matter physics community to predict self-assembly of patchy colloids.

This paper reviews some applications of the SAFT model in section 1.1 and describes modeling challenges for associating molecules in comparison with spectroscopic data in section 2. In sections 3 and 4, extensions of the SAFT association term to incorporate multiple bonding per association site and cooperative association are reviewed. These impact both associating molecules and patchy colloids. In section 5, recent density functional theory results for surfactants are reviewed. This is followed by conclusions.

\subsection{Applications of SAFT}

SAFT has been applied to natural gas dehydration and hydrate inhibition over the last decade. The main goal of gas dehydration is removing water to reduce pipeline corrosion and eliminate line blockage caused by hydrate formation. The water dew point should be below the lowest pipeline temperature to prevent free water formation. Hydrate formation may occur not only in pipelines, but also in cryogenic processes such as the production of liquefied natural gas (LNG) and of $\mathrm{C}_{2}+$ raw materials needed for the polymer industry. Consequently, the accurate 
determination of the water content of acid gas containing mixtures is essential for better process design and operation. As shown in Figure 1 (adapted from [26]), the perturbed chain form of the SAFT equation of state (PC-SAFT) $[10,11]$ accurately predicts the water content of natural gas mixtures across a wide range of temperatures and acid gas compositions.

SAFT has also found success in the upstream of the oil and gas industry, such as modeling of asphaltene phase behavior at reservoir conditions. Asphaltenes are the heaviest and most polarizable fraction of crude oil [28]. Asphaltenes are characterized by their solubility; they are completely soluble in aromatic solvents and insoluble in light paraffinic solvents at ambient conditions [29, 30]. Avoiding asphaltene precipitation and deposition is essential to maintaining an economic and efficient production process. As a result, understanding the phase behavior of asphaltenes is of great interest to the petroleum industry. Figure 2 (adapted from [31]) depicts the effect of gas injection on the destabilization of asphaltenes as predicted by PC-SAFT and the Cubic Plus Association (CPA) [16] equation of state. Asphaltenes are stable at high pressure, but can become unstable as the oil expands at low pressures. The pressure at which asphaltenes become unstable is the asphaltene onset pressure (AOP). Figure 2 shows data for the bubble point pressure (squares) and the AOP (circles). Both equations of state were fit to the AOP at 5\% gas injection and then used to predict the phase behavior for different injected gas amounts. They both can provide accurate predictions for the cases of low gas injection. In Figure 2, PC-SAFT better captures the increase in asphaltene instability at high gas injections. CPA predicts more stability for the asphaltene at higher gas injections.

Figure 3 (adapted from [33]) demonstrates one of the SAFT applications to the area of polymer solutions, in this case the phase diagram of the ternary system polystyrene (PS) + cyclohexane + carbon dioxide. The data measured by Bungert [34] of Wolfgang Arlt's group 
shows how the addition of $\mathrm{CO}_{2}$ affects the temperature and pressure dependent phase behavior of polystyrene in a solvent. In Figure 3, first consider the mixture with no carbon dioxide. The bubble point curve (essentially the vapor pressure curve for cyclohexane) is shown at the bottom. The nearly vertical phase boundary at about $20{ }^{\circ} \mathrm{C}$ is an upper critical solution temperature (UCST)-type boundary. Below this temperature, the system splits into two phases. As the temperature rises above this boundary, the system moves from a region of two liquid phases to a single liquid phase. There is another phase boundary as the temperature is increased at constant pressure; as the solvent expands, it becomes a poor solvent for the polymer producing a second liquid phase. This curve is a lower critical solution temperature (LCST)-type phase boundary. As the temperature is increased at constant pressure, the solvent expands and becomes a poor solvent for the polymer. Prediction of this type of phase boundary requires an equation of state because it is the compressibility of the system that causes the phase splitting. As seen in Figure 3, PC-SAFT accurately predicts that adding $\mathrm{CO}_{2}$ causes the bubble point curve to shift to higher pressures and shifting the LCST type phase boundary to higher pressures and lower temperatures. The merging of the UCSR and the LCST at approximately $21.25{ }^{\circ} \mathrm{C}$ is correctly predicted by the model although the model loses accuracy.

Finally, the incorporation of a polar term into the SAFT framework can be considered as one of the significant steps taken toward a better equation of state for the chemical industry $[12,13$, 36, 37]. Mixtures of non-polar and polar components such as ketones, ethers and esters are often difficult to model using a conventional non-polar equation of state due to large positive deviations from ideal solution behavior. Employing an equation of state that is based on perturbation theory offers the advantage of adding an explicit contribution due to polar interactions that accurately predicts the temperature and composition dependence. The 
significance of explicitly considering dipolar-dipolar interactions on the accurate determination of the phase behavior can be shown using the example illustrated in Figure 4 (adapted from [37]). The binary mixture of ethyl acetate $+\mathrm{n}$-heptane exhibits an azeotropic behavior, which is accurately reproduced by Polar PC-SAFT $[12,13]$ with the binary interaction parameter set to zero. On the other hand, the non-polar version of PC-SAFT was unable to capture the non-ideal behavior of the mixture using a zero or fitted binary interaction parameter.

\subsection{Challenges and opportunities}

Although the SAFT approach has been widely applied, opportunities remain to extend the range of the theory while improving the predictive ability of the model. Some of these topics are covered in this paper. Hydrogen bonding systems are commonly modeled with SAFT, but questions arise concerning the potential model to use for given systems. For example, SAFT models hydrogen bonds using electron donor and electron acceptor sites. Should the two lone pairs of electrons on an oxygen atom in an alcohol be modeled with a single electron donor site or two electron donor sites? Perhaps the user should not be forced to choose the number of association sites, but instead the theory could allow multiple bonding at association sites. Similar questions are raised for the solvation of an ion where the theory has the potential to predict the solvation number distribution. Further extension of the theory in the form of a density functional theory provides insight into how hydration of a surfactant affects interfacial properties and micelle formation. Each of these will be addressed below.

\section{Predicting hydrogen bonding distributions in associating fluids}

This section aims to examine the ability of PC-SAFT to accurately predict thermodynamics of mixtures containing associating components. Details of the model will be published shortly 
[39]. The accuracy of the theory for the extent of hydrogen bonding can be shown by comparing free monomer fractions predicted by the theory to spectroscopic data measured by Asprion et al. [40] and von Solms et al. [41]. In Figure 5, it can be seen that disagreement exists within the literature for the fraction of free ethanol monomers in n-hexane. As a result, molecular dynamic (MD) simulations were carried out in this work for further validation and comparison.

MD simulations of 1-alcohol + n-alkane binary mixtures were carried out using GROMACS 4.6.5 software package [42]. To parameterize 1-alcohol and n-alkane molecules, the OPLS-AA force field [43] was utilized. NVT and NPT simulations were first done for 5 ns to equilibrate the system and then another 5 ns NPT simulations were carried out for data collection and analysis. To compute the number of hydrogen-bonds formed in the system, the O-H... angle and distance between $\mathrm{O}$ and $\mathrm{O}$ criteria needed to be defined. A hydrogen bond was counted as formed only if $\mathrm{O}-\mathrm{H} \ldots \mathrm{O}$ angle is less than 30 degrees and the distance between $\mathrm{O}$ and $\mathrm{O}$ in two alcohol molecules is less than $3.7 \AA$. We also compare with Polar PC-SAFT fitted to pure component vapor pressure and saturated liquid density. Following Yarrison et al. [44] notations, self-association for alcohols was modeled with two association sites 2(1,1), one electron donor and one electron acceptor.

Both Polar PC-SAFT and MD simulation agree well with the monomer fractions measured by Asprion et al. [40] instead of that measured by von Solms et al. [41]. Figure 5 also illustrates the significance of considering dipole-dipole interactions in predicting the thermodynamics of self-association in alcohol containing systems. Specifically including polar interactions in the equation of state (Polar PC-SAFT) shifts the monomer fraction curve upward compared to PCSAFT (no long range polar contribution) which leads to better agreement with the spectroscopic 
data. As seen in Figure 6, Polar PC-SAFT was also able to accurately capture the temperature dependence of 1-butanol monomer fractions in n-hexane.

Interestingly, analysis of the IR experiments led Wilson et al. [45] to the conclusion that at a given temperature the extent of association is dependent on alcohol concentration while being independent of both the alcohol and the alkane chain length. This is predicted by SAFT but we can also predict the temperature dependence. From Wertheim's first order perturbation theory, the fraction of free monomers is dependent on the molar concentration of association sites, the mass density of the mixture through the pair correlation function and the temperature through the Mayer f-function. Although the equation of state parameters for the components shown in Table 1 show small differences in association energy, we assume that the association energy for all alcohols are approximately equal to that of pure ethanol. Assuming that the mass densities are not too different, SAFT predicts that all of the monomer fraction data should overlap when plotted against the molar concentration of association sites multiplied by the ratio of the Mayer f-function at a certain temperature to the Mayer f-function at $298 \mathrm{~K}$ as a reference [39]. As shown in Figure 7, the spectroscopic data for the monomer fractions from methanol to 1-hexanol alcohols in different solvents overlap.

Next, we examine the significance of bond cooperativity in predicting accurate hydrogen bonding distribution in a 1-alcohol + n-alkane system. To do so, Polar PC-SAFT and MD simulation were used by Fouad et al. [39] to predict the fraction of molecules bound in dimers for 1-butanol + n-hexane binary system. Results were also compared to scattered spectroscopic data measured by by Asprion et al. [40]. In reference to Figure 8, it can be seen that the overall agreement between the spectroscopic data and the MD simulations is good up to high alcohol concentrations. However, Figure 8 also shows that the Polar PC-SAFT equation of state over- 
predicts the fraction of molecules bound in dimers by nearly twice the amount. In agreement with previous FT-IR analysis done by Reilly et al. [46], over-predicting the fraction of molecules bound in dimers indicate that the strength of the second hydrogen bond formed by an alcohol is stronger than that of the first hydrogen bond. We believe that Figure 8 provides new evidence suggesting the importance of bond cooperativity in modeling the thermodynamics of hydrogen bonding in 1-alcohol $+\mathrm{n}$-alkane mixtures. The work done by Marshall and Chapman [47] as well as Sear and Jackson [48] on developing a re-summed thermodynamic perturbation theory for bond cooperativity can be possible approaches of incorporating such an effect in the current SAFT models.

\section{Beyond Wertheim's first order perturbation theory}

As shown above and in Fouad et al. [39], SAFT results depend on the number of association sites chosen and the effect of bond cooperativity is not captured. Wertheim's first order perturbation theory has limitations on modeling associating fluids [4-7]. In first order theory, the

sites are considered saturated after forming only a single bond and a site must break a bond before the site is available to bond again. . Cooperative association or variation in bonding energies along a cluster of associated beads is another effect which is not captured by this theory. In this section we review some the recent advances beyond first order theory.

\subsection{Bonding more than once at a site}

Depending on the range of interaction of an association site, a limited number of bonds can form before the site is saturated. In Wertheim's first order theory, the range of association sites are assumed to be small enough that particle repulsions prevent formation of more than one bond at an association site; however, this steric effect is not true for all systems. For example, the 
question of whether to model the two lone pairs of electrons of an oxygen atom in water or alcohols as a single association site or two sites is still unanswered because, depending on the condition of the system, either may give better results. In addition, the size of a patch in patchy colloidal systems [49] can be big enough to form more than one bond, hence multi-bonding must be considered in the model. Therefore, it is helpful if the site is modeled appropriately such that according to the conditions of the system it forms one or more bonds.

Recently, researchers made tremendous progress in modeling sites that can bond multiple times [50-53]. Marshall and Chapman [52, 53] extended Wertheim's first order theory for single site fluids to allow for multiple bonding and thus formation of chains and rings. Such an approach shows promise toward predicting extents of association due to steric effects. Similar ideas are used to incorporate solvation of ions as described in section 4.

\subsection{Bond cooperativity effect}

Hydrogen bonding molecules sometimes show energy variations through bonding more than once in a cluster. For example; the strength of association in hydrogen fluoride (HF) changes along the cluster of associated HF's [54]. This effect can carry an impact over the extent of association inside the fluid, because the possible difference between bonding energies can lead to forming either longer or shorter chains or rings corresponding to more or less association.

In the first order limit, there is only a single Mayer f-function and it is not possible to include any variations in bonding energy through a cluster. Sear and Jackson [48] were the first to suggest a solution for bond cooperativity in a two-site fluid, where the energy of the first bond is different than the other bonds. Later, Marshall and Chapman [47] developed a new approach to model the same system using infinite order perturbation theory. Marshall et al. [55] also, 
combined the bond cooperativity and bond angle effects and developed a new equation of state dependent on both. We have developed a new approach to capture this effect using Wertheim's two-density formalism, in which each species is identified with a density factor that shows whether it is bonded or not, in the limit of infinite order for a two-site Lenard Jones (LJ) fluid with a large bond angle that avoids steric hindrance effects. In our model only unlike sites are allowed to associate, and due to what we chose for the bond angle, only formation of linear chains of any length is predicted. Figure 9 shows a schematic view of a chain of associated beads where the energy of the first bond formed is different than other bonds. Details of the theory will be published shortly.

In Figure 10, we compare the theory results with MC predictions for fluids with a fixed reduced density $\left(\rho \sigma^{3}\right)$, and association energies $\left(\varepsilon_{A B}^{(1)} / k_{B} T, \varepsilon_{A B}^{(2)} / k_{B} T\right)$, while the LJ energy $\left(\varepsilon_{L J} / k_{B} T\right)$ is increasing. The results are in an excellent agreement with simulation. According to this figure, in a positive cooperativity $\left(\varepsilon^{(1)}<\varepsilon^{(2)}\right)$ increasing LJ energy results in breaking the bonds. Because as LJ energy grows beads tend to locate themselves at a distance from each other at which they experience the maximum LJ attraction. Here we set the association critical distance to 1.05 sigma which is smaller than the LJ minimum energy distance of 1.12 sigma. Therefore, by maximizing LJ attraction between molecules fewer molecules are available in the range of the hydrogen bonding interaction.

\section{Incorporating multi-body effects for modeling electrolyte systems}

Short range structuring is important in determining the mean density of electrolyte systems. Ion solvent association or ion-solvation describes arrangement of water or solvent molecules around an ion. Various versions of SAFT that have been used to model electrolyte systems [56- 
61], both primitive and non-primitive, use ion-solvent association to describe ion-solvation. More recently, such ion-solvent association has been used to inform dielectric constant calculations in salt solutions [62]. Wertheim's perturbation theory [4-7], which forms the basis for different versions of SAFT and CPA [16], uses the pair-correlation information from the reference system to model the behavior of fluids with short-range directional interactions. However, Wertheim's theory falls short of capturing the multi-body effects that are observed in solute-solvent interactions; therefore the approach does not hold for a spherically attractive solute in the presence of solvent molecules with short-range directional interactions.

To incorporate multi-body effects in SAFT when the association potential of the solute is assumed to be spherically symmetric, as opposed to directional, Marshall and Chapman [63, 64] developed a theory based on Wertheim's multi-density [6] formalism for multi-site associating fluids. The system is defined as a self-assembling mixture of patchy molecules with directional interactions (treated with standard first order thermodynamic perturbation theory (TPT1) [5]) and solute with spherically symmetric attractions. In our equation of state based model for electrolyte solutions, we have incorporated a spherically symmetric short ranged association potential on the ion that can associate with directional patchy sites on the solvent to describe hydration of the ion. This association scheme between ion and solvent is modeled using Marshall-Chapman theory [63] and calculates average hydration of ion within the theory as opposed to earlier approaches using fixed number of sites for ions within SAFT formalism for electrolyte systems. Long-range effects are included using an integral equation method (semi restricted non-primitive MSA [65]). With this novel ion solvation approach, characteristic information can be retrieved such as how the hydration number distribution varies with concentration and temperature. Also for primitive 
models to incorporate the dielectric constant variation, changes in hydration numbers with concentration, which are input to the model, are inherently accounted in the theory.

For electrolyte systems, it is important to study their behavior in mixtures of different salts and solvents. Hence, to obtain physically meaningful ion-specific transferrable parameters, we have utilized ion-hydration information at infinite dilution obtained from MD simulations along with activity coefficient data at $298.15 \mathrm{~K}$ for the concentration range. We find that this novel approach can represent the hydration distribution behavior in electrolyte systems better than specifying a fixed number of hydration sites on the ions. For concentrated solutions, we compared the densities of the solution with experimental data. It was observed that the density predictions over a wide range of temperatures and concentration were in excellent agreement with the experimentally $[66,67]$ observed values (see Figure 11). Agreement is better than what has been reported in the literature for a model with as few parameters. Furthermore, we find that the parameters are transferrable to model other salts as well. While, excellent agreement for densities is obtained, predicting activity coefficient at high temperature is still a challenge. Further details and refinements will be reported in future publications.

\section{Application of iSAFT to micelle formation}

In previous sections, we mainly focused on the applications of Statistical Associating Fluid Theory or SAFT for homogeneous systems. In this section, we will focus on inhomogeneous systems with the presence of surfactants and the application of interfacial Statistical Associating Fluid Theory or iSAFT $[25,68]$ for such systems. iSAFT has been applied to block copolymers $[69,70]$, polymer brushes [71-73], associating molecules [74-78], and surfactants [79, 80]. Here we consider micelle formation. 
Surfactants are amphiphilic molecules consisting of two chemically distinct components, hydrophobic tail and hydrophilic head. Due to this unique molecular nature, surfactants can selfassemble at interfaces to reduce interfacial tension or aggregate in solution to form micelles [81]. Surfactants lower the surface free energy of the system by reducing the unfavorable contact between two phases. Due to their capability of altering interfacial properties, surfactants are widely used in areas ranging from personal care products to industrial processes including enhanced oil recovery [82]. Understanding the relations between surfactant architecture and properties of surfactant is of vital importance in these applications. The theoretical tool we use to study the structure and properties of these systems is the interfacial Statistical Associating Fluid Theory or iSAFT $[25,68]$. iSAFT was previously used to explore the parameter space of structure-property relationships of surfactants at liquid-liquid interfaces by Emborsky et al. [79] and study the behavior of amphiphilic molecules with associating sites at water/oil interface by Marshall et al. [78, 80]. The theory has been shown to be able to produce Traube's rule [83] and reveal the structure and properties of interfaces in good agreement with molecular simulations [77].

Here, we review recent work on the study of micellization of surfactant systems based on iSAFT density functional theory. By revising the iSAFT model developed by Jain et al. [68] to the form in spherical coordinates, the micelle formation can be readily modeled. A detailed description of the model and results will be subject of a future paper. We consider a model surfactant/water mixture, and we assume the micelle formed has a spherical shape [84]. The water is modeled as a sphere with four short ranged associating sites, two electron donor sites and two electron acceptor sites. The model surfactant is polyethylene glycol monooctyl ether or $\mathrm{CnEm}$, and it is modeled as a combination of a chain of non-associating segments as surfactant 
tail and a chain of associating segments with two associating sites (electron donors) on each segment which can form hydrogen bonds with water molecules. The critical micelle concentration, which is defined as the concentration of surfactants above which micelles form, is determined by comparing the grand potentials of systems with or without the formation of micelle at the same bulk surfactant concentration. Here, we compare the grand potential because we are using the grand canonical ensemble with the chemical potential, volume and temperature fixed at a specific bulk surfactant concentration. When the grand potential of the system with a micelle is lower than that without a micelle, the micellar phase is stable and the critical micelle concentration is reached. The grand potential of $\mathrm{H}_{5} \mathrm{~T}_{4}$ or $\mathrm{C}_{8} \mathrm{E}_{5}$ surfactant is plotted in Figure 12 . We can see that at lower bulk surfactant concentration, the grand potential of the system with the micelle is greater than that without the micelle. This means that the micellar phase is not stable and hence the critical micelle concentration is not reached. The critical micelle concentration is reached when the grand potential of system with micelle is equal to that of system without micelle.

At the critical micelle concentration, the structure of micelle is plotted in Figure 13. We can observe that surfactant tails aggregate forming a hydrophobic core. Surfactant heads are hydrated by water molecules forming the outer shell of the micelle, and this outer shell prevents the direct contact between hydrophobic and hydrophilic elements. Since the hydration of surfactant heads by water molecules stabilizes the micelle, understanding hydrogen bonding is important in modeling micellar systems.

To conclude, the iSAFT density functional theory has been used to determine the critical micelle concentration and structure of micelle in a model surfactant/water mixture. The model 
may be further extended to study the micellar solubilization or encapsulation, which has significant applications in the fields of drug delivery, ultrafiltration, etc $[85,86]$.

\section{Conclusions}

In this paper, we have reviewed material presented during the SAFT 2015 conference in honor of the 25th anniversary of the SAFT equation of state. The SAFT equation of state provides a platform for engineers in industry and researchers in academia to predict properties of complex fluids mixtures showing large size asymmetry and association. Beyond molecular fluids, the model has been applied with equal success to patchy colloids. Example applications of the SAFT model were presented in this manuscript. In alcohol mixtures, the SAFT model accurately predicts that multiple spectroscopic data sets can collapse onto a single curve. We also showed challenges in comparing the association model to spectroscopic data for alcohol mixtures that indicate a need to include cooperative association effects. Extension of the theory to allow multiple bonds per association site and cooperative association are reviewed to model alcohol, electrolyte, and patchy colloid systems. Comparisons of these recently developed models with molecular simulation results opportunities for new applications. These theories for homogeneous fluids are also applicable to inhomogeneous systems through a density functional theory. To demonstrate this, an example application of this density functional theory for surfactants at the critical micelle concentration was presented.

\section{Acknowledgement}

W.A.F. gratefully acknowledges the Abu Dhabi National Oil Company (ADNOC) for financial support through a Ph.D. scholarship. L.W. gratefully acknowledges Rice University Consortium for Processes in Porous Media for financial support. A.H. gratefully acknowledges the financial 
support from the Robert A. Welch Foundation (Grant No. C-1241). A.B. gratefully acknowledges RPSEA / DOE 10121-4204-01 for funding the project on "Corrosion and Scale at Extreme Temperature and Pressure". 


\section{References}

[1] W.G. Chapman, G. Jackson, K.E. Gubbins, Phase equilibria of associating fluids: chain molecules with multiple bonding sites, Mol. Phys. 65 (1988) 1057-1079.

[2] W.G. Chapman, K.E. Gubbins, G. Jackson, M. Radosz, SAFT: Equation-of-state solution model for associating fluids, Fluid Phase Equilib. 52 (1989) 31-38.

[3] W.G. Chapman, K.E. Gubbins, G. Jackson, M. Radosz, New reference equation of state for associating liquids, Ind. Eng. Chem. Res. 29 (1990) 1709-1721.

[4] M.S. Wertheim, Fluids with highly directional attractive forces. I. Statistical thermodynamics, J. Stat. Phys. 35 (1984) 19-34.

[5] M.S. Wertheim, Fluids with highly directional attractive forces. II. Thermodynamic perturbation theory and integral equations, J. Stat. Phys. 35 (1984) 35-47.

[6] M.S. Wertheim, Fluids with highly directional attractive forces. III. Multiple attraction sites, J. Stat. Phys. 42 (1986) 459-476.

[7] M.S. Wertheim, Fluids with highly directional attractive forces. IV. Equilibrium polymerization, J. Stat. Phys. 42 (1986) 477-492.

[8] S.H. Huang, M. Radosz, Equation of state for small, large, polydisperse, and associating molecules, Ind. Eng. Chem. Res. 29 (1990) 2284-2294.

[9] S.H. Huang, M. Radosz, Equation of state for small, large, polydisperse, and associating molecules: extension to fluid mixtures, Ind. Eng. Chem. Res. 30 (1991) 1994-2005.

[10] J. Gross, G. Sadowski, Perturbed-chain SAFT: An equation of state based on a perturbation theory for chain molecules, Ind. Eng. Chem. Res. 40 (2001) 1244-1260.

[11] J. Gross, G. Sadowski, Application of the perturbed-chain SAFT equation of state to associating systems, Ind. Eng. Chem. Res. 41 (2002) 5510-5515. 
[12] P.K. Jog, W.G. Chapman, Application of Wertheim's thermodynamic perturbation theory to dipolar hard sphere chains, Mol. Phys. 97 (1999) 307-319.

[13] P.K. Jog, S.G. Sauer, J. Blaesing, W.G. Chapman, Application of dipolar chain theory to the phase behavior of polar fluids and mixtures, Ind. Eng. Chem. Res. 40 (2001) 4641-4648.

[14] N. von Solms, M.L. Michelsen, G.M. Kontogeorgis, Computational and physical performance of a modified PC-SAFT equation of state for highly asymmetric and associating mixtures, Ind. Eng. Chem. Res. 42 (2003) 1098-1105.

[15] S. Tamouza, J.P. Passarello, P. Tobaly, J.C. de Hemptinne, Group contribution method with SAFT EOS applied to vapor liquid equilibria of various hydrocarbon series, Fluid Phase Equilib. 222 (2004) 67-76.

[16] G.M. Kontogeorgis, E.C. Voutsas, I.V. Yakoumis, D.P. Tassios, An equation of state for associating fluids, Ind. Eng. Chem. Res. 35 (1996) 4310-4318.

[17] A. Gil-Villegas, A. Galindo, P.J. Whitehead, S.J. Mills, G. Jackson, A.N. Burgess, Statistical associating fluid theory for chain molecules with attractive potentials of variable range, J. Chem. Phys. 106 (1997) 4168-4186.

[18] F.J. Blas, L.F. Vega, Thermodynamic behaviour of homonuclear and heteronuclear Lennard-Jones chains with association sites from simulation and theory, Mol. Phys. 92 (1997) $135-150$.

[19] A. Lymperiadis, C.S. Adjiman, A. Galindo, G. Jackson, A group contribution method for associating chain molecules based on the statistical associating fluid theory (SAFT- $\gamma$ ), J. Chem. Phys. 127 (2007) 234903. 
[20] A. Lymperiadis, C.S. Adjiman, G. Jackson, A. Galindo, A generalisation of the SAFT-group contribution method for groups comprising multiple spherical segments, Fluid Phase Equilib. 274 (2008) 85-104.

[21] V. Papaioannou, T. Lafitte, C. Avendaño, C.S. Adjiman, G. Jackson, E.A. Müller, A. Galindo, Group contribution methodology based on the statistical associating fluid theory for heteronuclear molecules formed from Mie segments, J. Chem. Phys. 140 (2014) 054107.

[22] W.G. Chapman, Prediction of the thermodynamic properties of associating Lennard-Jones fluids: Theory and simulation, J. Chem. Phys. 93 (1990) 4299-4304.

[23] J.M. Stevenson, W.A. Fouad, D. Shalloway, D. Usher, J. Lunine, W.G. Chapman, P. Clancy, Solvation of nitrogen compounds in Titan's seas, precipitates, and atmosphere, Icarus $256(2015) 1-12$.

[24] S. Jain, A. Dominik, W.G. Chapman, Modified interfacial statistical associating fluid theory: A perturbation density functional theory for inhomogeneous complex fluids, J. Chem. Phys. 127 (2007) 244904.

[25] S. Tripathi, W.G. Chapman, Microstructure of inhomogeneous polyatomic mixtures from a density functional formalism for atomic mixtures, J. Chem. Phys. 122 (2005) 094506.

[26] W.A. Fouad, M. Yarrison, K.Y. Song, K.R. Cox, W.G. Chapman, High pressure measurements and molecular modeling of the water content of acid gas containing mixtures, AlChE J. 61 (2015) 3038-3052.

[27] S.C. Sharma, Equilibrium water content of gaseous mixtures, in, The University of Oklahoma., 1969. 
[28] F.M. Vargas, D.L. Gonzalez, J.L. Creek, J. Wang, J. Buckley, G.J. Hirasaki, W.G. Chapman, Development of a General Method for Modeling Asphaltene Stability, Energy Fuels 23 (2009) 1147-1154.

[29] D.L. Mitchell, J.G. Speight, The solubility of asphaltenes in hydrocarbon solvents, Fuel 52 (1973) 149-152.

[30] T.F. Yen, J.G. Erdman, S.S. Pollack, Investigation of the structure of petroleum asphaltenes by X-ray diffraction, Analytical Chemistry 33 (1961) 1587-1594.

[31] A.A. AlHammadi, F.M. Vargas, W.G. Chapman, Comparison of CPA and PC-SAFT Methods for Modeling Asphaltene Phase Behavior and PVT Properties, Energy Fuels 29 (2015) 2864-2875.

[32] S.R. Panuganti, F.M. Vargas, D.L. Gonzalez, A.S. Kurup, W.G. Chapman, PC-SAFT characterization of crude oils and modeling of asphaltene phase behavior, Fuel 93 (2012) 658669.

[33] F.M. Vargas, D.L. Gonzalez, G.J. Hirasaki, W.G. Chapman, Modeling Asphaltene Phase Behavior in Crude Oil Systems Using the Perturbed Chain Form of the Statistical Associating Fluid Theory (PC-SAFT) Equation of State, Energy Fuels 23 (2009) 1140-1146.

[34] B. Bungert, Komplexe Phasengleichgewichte von Polymerlösungen, in, Technische Universität Berlin, Germany, 1998.

[35] S. Saeki, N. Kuwahara, S. Konno, M. Kaneko', Upper and lower critical solution temperatures in polystyrene solutions, Macromolecules 6 (1973) 246-250.

[36] S.G. Sauer, W.G. Chapman, A parametric study of dipolar chain theory with applications to ketone mixtures, Ind. Eng. Chem. Res. 42 (2003) 5687-5696. 
[37] A. Dominik, W.G. Chapman, M. Kleiner, G. Sadowski, Modeling of polar systems with the perturbed-chain SAFT equation of state. Investigation of the performance of two polar terms, Ind. Eng. Chem. Res. 44 (2005) 6928-6938.

[38] G.S. Shealy, S.I. Sandler, Vapor-liquid equilibria of $\{\mathrm{xCH} 3 \mathrm{CH}(\mathrm{CH} 3) \mathrm{CHO}+(1-\mathrm{x}) \mathrm{C} 7 \mathrm{H}$ $16\}(1)$ and $\{\mathrm{xCH} 3 \mathrm{CO} 2 \mathrm{C} 2 \mathrm{H} 5+(1-\mathrm{x}) \mathrm{C} 7 \mathrm{H} 16\}(1), \mathrm{J}$. Chem. Thermodyn. 17 (1985) 143-150.

[39] W.A. Fouad, L. Wang, A. Haghmoradi, S.K. Gupta, W.G. Chapman, Understanding the Thermodynamics of Hydrogen Bonding in Alcohol Containing Mixtures: Self Association, J. Phys. Chem. B 119 (2015) 14086-14101.

[40] N. Asprion, H. Hasse, G. Maurer, FT-IR spectroscopic investigations of hydrogen bonding in alcohol-hydrocarbon solutions, Fluid Phase Equilib. 186 (2001) 1-25.

[41] N. von Solms, L. Jensen, J.L. Kofod, M.L. Michelsen, G.M. Kontogeorgis, Measurement and modelling of hydrogen bonding in 1-alkanol+n-alkane binary mixtures, Fluid Phase Equilib. 261 (2007) 272-280.

[42] B. Hess, C. Kutzner, D. Van Der Spoel, E. Lindahl, GROMACS 4: Algorithms for highly efficient, load-balanced, and scalable molecular simulation, J. Chem. Theory Comput. 4 (2008) 435-447.

[43] W.L. Jorgensen, D.S. Maxwell, J. Tirado-Rives, Development and testing of the OPLS allatom force field on conformational energetics and properties of organic liquids, J. Am. Chem. Soc. 118 (1996) 11225-11236.

[44] M. Yarrison, W.G. Chapman, A systematic study of methanol + n-alkane vapor-liquid and liquid-liquid equilibria using the CK-SAFT and PC-SAFT equations of state, Fluid Phase Equilib. 226 (2004) 195-205. 
[45] L. Wilson, R.B. de Alencastro, C. Sandorfy, Hydrogen bonding of n-alcohols of different chain lengths, Can. J. Chem. 63 (1985) 40-45.

[46] J.T. Reilly, A. Thomas, A.R. Gibson, C.Y. Luebehusen, M.D. Donohue, Analysis of the Self-Association of Aliphatic Alcohols Using Fourier Transform Infrared (FT-IR) Spectroscopy, Ind. Eng. Chem. Res. 52 (2013) 14456-14462.

[47] B.D. Marshall, W.G. Chapman, Resummed thermodynamic perturbation theory for bond cooperativity in associating fluids, J. Chem. Phys. 139 (2013) 214106.

[48] R.P. Sear, G. Jackson, Thermodynamic perturbation theory for association with bond cooperativity, J. Chem. Phys. 105 (1996) 1113-1120.

[49] Y.F. Wang, Y. Wang, D.R. Breed, V.N. Manoharan, L. Feng, A.D. Hollingsworth, M. Weck, D.J. Pine, Colloids with valence and specific directional bonding, Nature 491 (2012) 5155.

[50] Y.V. Kalyuzhnyi, H. Docherty, P.T. Cummings, Resummed thermodynamic perturbation theory for central force associating potential: One-patch model, J. Chem. Phys. 133 (2010) 044502.

[51] Y.V. Kalyuzhnyi, H. Docherty, P.T. Cummings, Resummed thermodynamic perturbation theory for central force associating potential. Multi-patch models, J. Chem. Phys. 135 (2011) 014501.

[52] B.D. Marshall, D. Ballal, W.G. Chapman, Wertheim's association theory applied to one site patchy colloids: Beyond the single bonding condition, J. Chem. Phys. 137 (2012) 104909.

[53] B.D. Marshall, W.G. Chapman, A density functional theory for patchy colloids based on Wertheim's association theory: Beyond the single bonding condition, J. Chem. Phys. 138 (2013) 044901. 
[54] L. Rincón, R. Almeida, D. Garcia-Aldea, H. Diez y Riega, Hydrogen bond cooperativity and electron delocalization in hydrogen fluoride clusters, J. Chem. Phys. 114 (2001) 5552-5561. [55] B.D. Marshall, A. Haghmoradi, W.G. Chapman, Resummed thermodynamic perturbation theory for bond cooperativity in associating fluids with small bond angles: Effects of steric hindrance and ring formation, J. Chem. Phys. 140 (2014) 164101.

[56] Y. Liu, Z. Li, J. Mi, C. Zhong, Modeling of aqueous electrolyte solutions based on primitive and first-order mean spherical approximation, Ind. Eng. Chem. Res. 47 (2008) 1695-1701.

[57] Z. Liu, W. Wang, Y. Li, An equation of state for electrolyte solutions by a combination of low-density expansion of non-primitive mean spherical approximation and statistical associating fluid theory, Fluid Phase Equilib. 227 (2005) 147-156.

[58] Z.-P. Liu, Y.-G. Li, J.-F. Lu, Low-Density Expansion of the Solution of Mean Spherical Approximation for Ion-Dipole Mixtures, J. Phys. Chem. B 106 (2002) 5266-5274.

[59] B.-S. Lee, K.-C. Kim, Modeling of aqueous electrolyte solutions based on perturbed-chain statistical associating fluid theory incorporated with primitive mean spherical approximation, Korean J. Chem. Eng. 26 (2009) 1733-1747.

[60] J. Rozmus, J.-C. de Hemptinne, A. Galindo, S. Dufal, P. Mougin, Modeling of strong electrolytes with ePPC-SAFT up to high temperatures, Ind. Eng. Chem. Res. 52 (2013) 99799994.

[61] S. Herzog, J. Gross, W. Arlt, Equation of state for aqueous electrolyte systems based on the semirestricted non-primitive mean spherical approximation, Fluid Phase Equilib. 297 (2010) 2333.

[62] B. Maribo-Mogensen, G.M. Kontogeorgis, K. Thomsen, Modeling of Dielectric Properties of Aqueous Salt Solutions with an Equation of State, J. Phys. Chem. B 117 (2013) 10523-10533. 
[63] B.D. Marshall, W.G. Chapman, Thermodynamic perturbation theory for self assembling mixtures of multi-patch colloids and colloids with spherically symmetric attractions, Soft Matter 9 (2013) 11346-11356.

[64] B.D. Marshall, W.G. Chapman, Molecular theory for self assembling mixtures of patchy colloids and colloids with spherically symmetric attractions: The single patch case, J. Chem. Phys. 139 (2013) 104904.

[65] D. Wei, L. Blum, The mean spherical approximation for an arbitrary mixture of ions in a dipolar solvent: Approximate solution, pair correlation functions, and thermodynamics, J. Chem. Phys. 87 (1987) 2999-3007.

[66] D.G. Archer, Thermodynamic Properties of the $\mathrm{NaBr}+\mathrm{H} 2 \mathrm{O}$ System, Journal of Physical and Chemical Reference Data 20 (1991) 509-555.

[67] K.S. Pitzer, J.C. Peiper, R.H. Busey, Thermodynamic properties of aqueous sodium chloride solutions, Journal of Physical and Chemical Reference Data 13 (1984) 1-102.

[68] S. Jain, P. Jog, J. Weinhold, R. Srivastava, W.G. Chapman, Modified interfacial statistical associating fluid theory: Application to tethered polymer chains, J. Chem. Phys. 128 (2008) 154910.

[69] S. Jain, W.G. Chapman, Effect of confinement on the ordering of symmetric diblock copolymers: application of interfacial statistical associating fluid theory, Mol. Phys. 107 (2009) $1-17$.

[70] Z. Feng, W.G. Chapman, Revisited Block Copolymer/Nanoparticle Composites: Extension of Interfacial Statistical Associating Fluid Theory, Macromolecules 45 (2012) 6658-6668.

[71] K. Gong, W.G. Chapman, Solvent response of mixed polymer brushes, J. Chem. Phys. 135 (2011) 214901. 
[72] K. Gong, B.D. Marshall, W.G. Chapman, Response behavior of diblock copolymer brushes in explicit solvent, J. Chem. Phys. 137 (2012) 154904.

[73] K. Gong, B.D. Marshall, W.G. Chapman, Modeling lower critical solution temperature behavior of associating polymer brushes with classical density functional theory, J. Chem. Phys. 139 (2013) 094904.

[74] D. Ballal, W.G. Chapman, Competition Between Intra and Intermolecular Association of Chain Molecules with Water-like Solvent, J. Phys. Chem. B 119 (2015) 6792-6802.

[75] D. Ballal, W.G. Chapman, Hydrophobic and hydrophilic interactions in aqueous mixtures of alcohols at a hydrophobic surface, J. Chem. Phys. 139 (2013) 114706.

[76] A. Bymaster, W.G. Chapman, An i SAFT density functional theory for associating polyatomic molecules, J. Phys. Chem. B 114 (2010) 12298-12307.

[77] B.D. Marshall, K.R. Cox, W.G. Chapman, A classical density functional theory study of the neat n-alkane/water interface, J. Phys. Chem. C 116 (2012) 17641-17649.

[78] B.D. Marshall, K.R. Cox, W.G. Chapman, Supramolecular assembly and surfactant behavior of triblock rod-coil amphiphiles at liquid interfaces using classical density functional theory, Soft Matter 8 (2012) 7415-7425.

[79] C.P. Emborsky, K.R. Cox, W.G. Chapman, Exploring parameter space effects on structureproperty relationships of surfactants at liquid-liquid interfaces, J. Chem. Phys. 135 (2011) 084708 .

[80] B.D. Marshall, C. Emborsky, K. Cox, W.G. Chapman, Effect of bond rigidity and molecular structure on the self-assembly of amphiphilic molecules using second-order classical density functional theory, J. Phys. Chem. B 116 (2012) 2730-2738. 
[81] C.A. Miller, P. Neogi, Interfacial phenomena: equilibrium and dynamic effects, CRC Press, 2007.

[82] G.J. Hirasaki, C.A. Miller, M. Puerto, Recent advances in surfactant EOR, in: SPE Annual Technical Conference and Exhibition, Society of Petroleum Engineers, 2008.

[83] J. Traube, Ueber die Capillaritätsconstanten organischer Stoffe in wässerigen Lösungen, Justus Liebigs Ann. Chem. 265 (1891) 27-55.

[84] R. Rajagopalan, P.C. Hiemenz, Principles of colloid and surface chemistry, Marcel Dekker, New-York, 3e édition, ISBN 08247 (1997) 8.

[85] P.H. Elworthy, A.T. Florence, C.B. Macfarlane, Solubilization by surface-active agents and its applications in chemistry and the biological sciences, 1968.

[86] D. Attwood, Surfactant systems: their chemistry, pharmacy and biology, Springer Science \& Business Media, 2012. 
Table 1 Pure component parameters used in this work.

\begin{tabular}{|c|c|c|c|c|c|c|c|c|c|}
\hline Component & $m_{i}$ & $\begin{array}{c}\sigma_{i} \\
(\AA)\end{array}$ & $\begin{array}{c}\varepsilon_{i} / k \\
(\mathrm{~K})\end{array}$ & $\begin{array}{c}\varepsilon^{A_{i} B_{i}} / k \\
(\mathrm{~K})\end{array}$ & $\kappa^{A_{i} B_{i}}$ & $\begin{array}{c}\mu_{i} \\
(\mathrm{D})\end{array}$ & $m_{i} x_{p_{i}}$ & $\begin{array}{c}\text { AAD\% } \% \\
p^{\text {sat }}\end{array}$ & $\begin{array}{c}\text { AAD\% } \% \\
\rho^{\text {sat }}\end{array}$ \\
\hline ethanol & 2.000 & 3.428 & 230 & 2515.569 & 0.0179 & 1.70 & 0.50 & 0.93 & 1.57 \\
\hline 1-butanol & 3.000 & 3.481 & 237 & 2535.788 & 0.0125 & 1.70 & 0.50 & 3.48 & 0.25 \\
\hline n-hexane & 3.058 & 3.798 & 236.77 & & & & & 0.31 & 0.76 \\
\hline
\end{tabular}



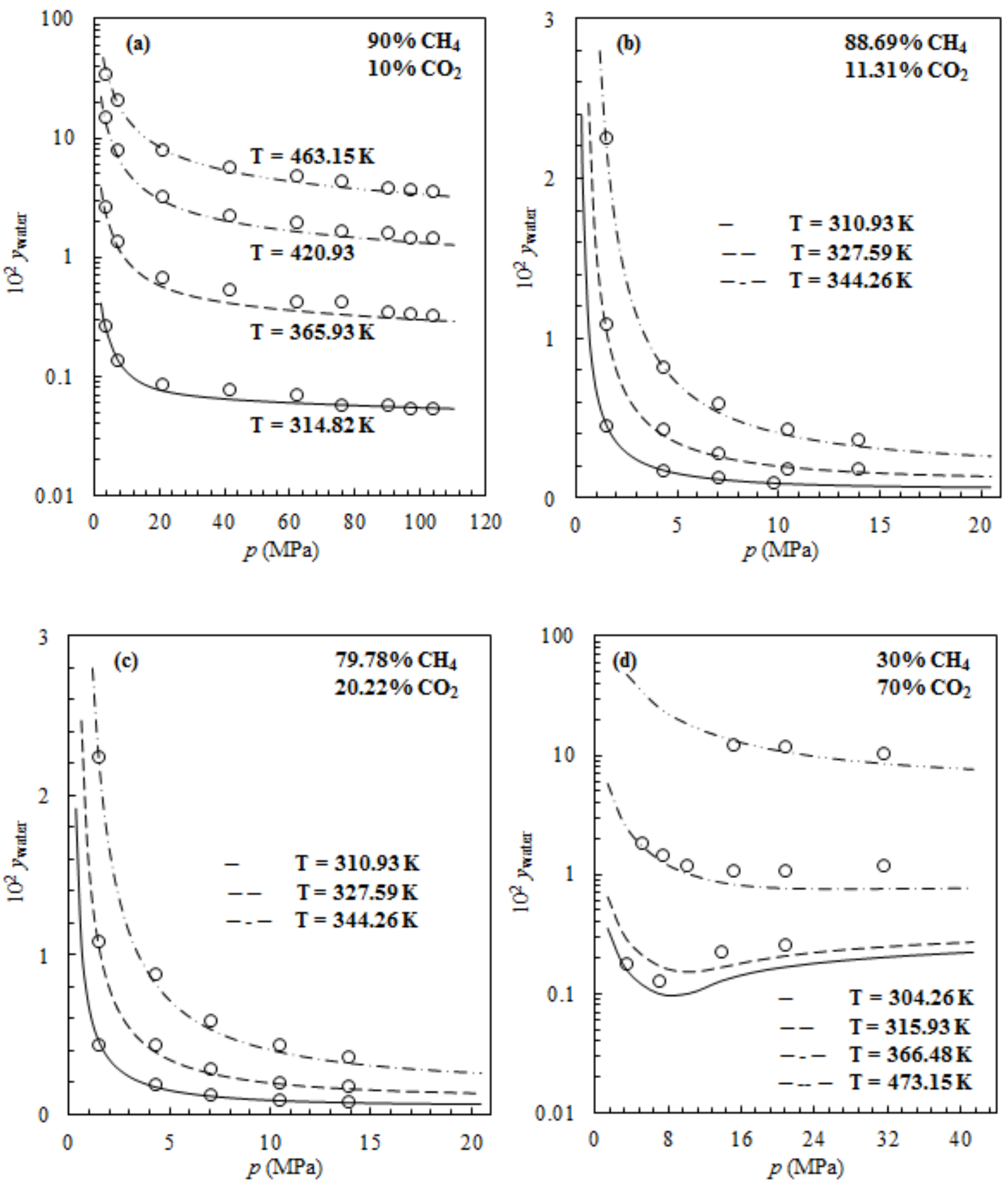

Figure 1 Water content of methane + carbon dioxide mixture as a function of temperature and pressure. (o): experimental data; (-): PC-SAFT predictions. (a): experimental data by Fouad et al. [26]; (b): experimental data by Sharma [27] for gaseous mixture of $88.69 \mathrm{~mol} . \% \mathrm{CH}_{4}$ and 11.31 mol. \% $\mathrm{CO}_{2}$; (c): experimental data by Sharma [27] for gaseous mixture of $79.78 \mathrm{~mol}$ \% $\mathrm{CH}_{4}$ and 20.22 mol. \% $\mathrm{CO}_{2}$; (d): experimental data by Fouad et al. (adapted from [26]). 

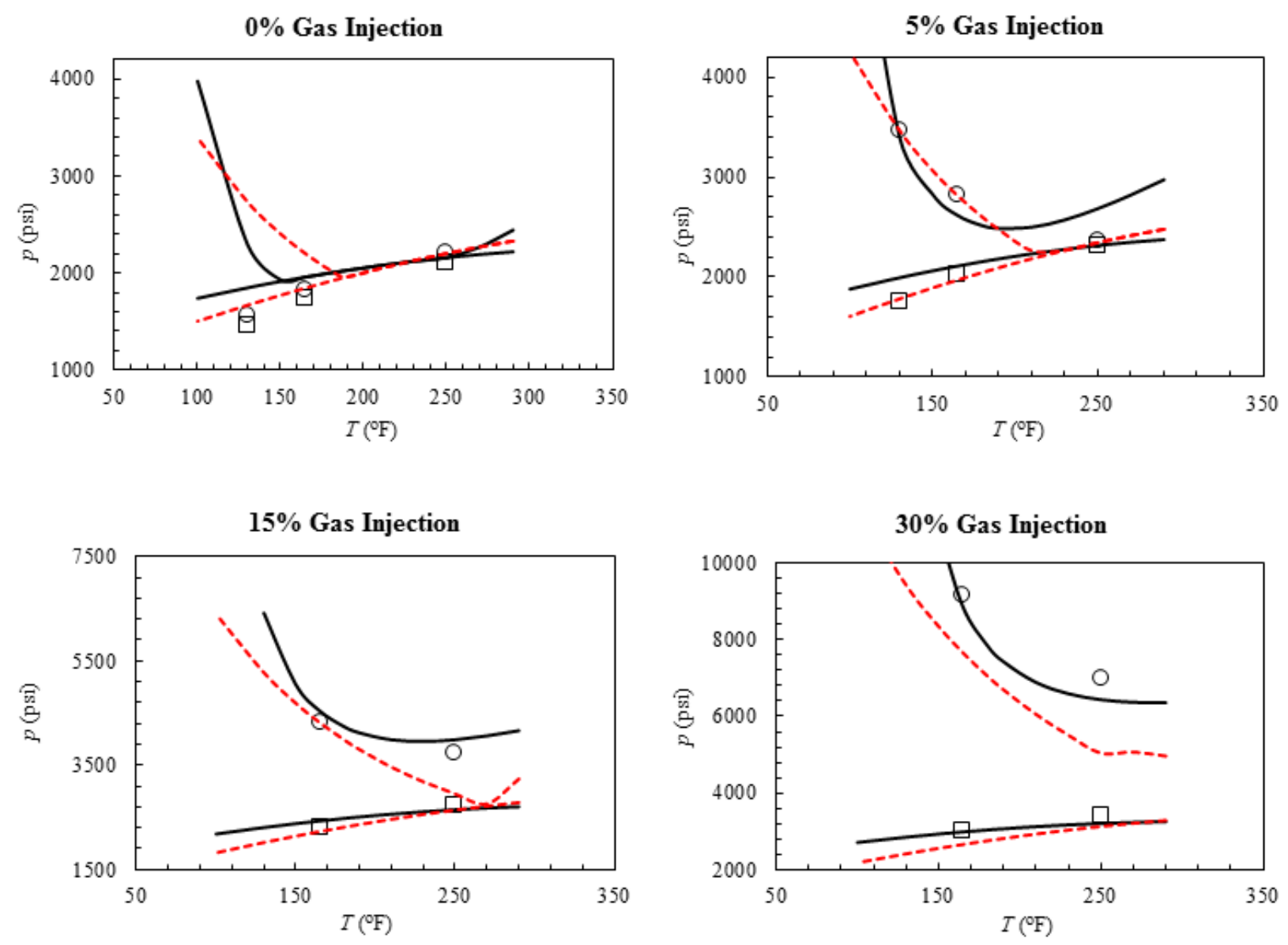

Figure 2 PC-SAFT and CPA characterized oil prediction for crude A after estimating the parameters for 5 mol. \% of gas injection data. (o): experimental AOP; ( $\square$ ): experimental bubble pressure [32]. Solid black curve (-): PC-SAFT; dashed red curve (- ): CPA. Injected gas composition (mol. \%): $\mathrm{N}_{2}-0.4 \%, \mathrm{CO}_{2}-3.9 \%, \mathrm{C}_{1}-71.4 \%, \mathrm{C}_{2}-12 \%, \mathrm{C}_{3}-7.2 \%$, heavy gas-5.1\% (adapted from [31]). 


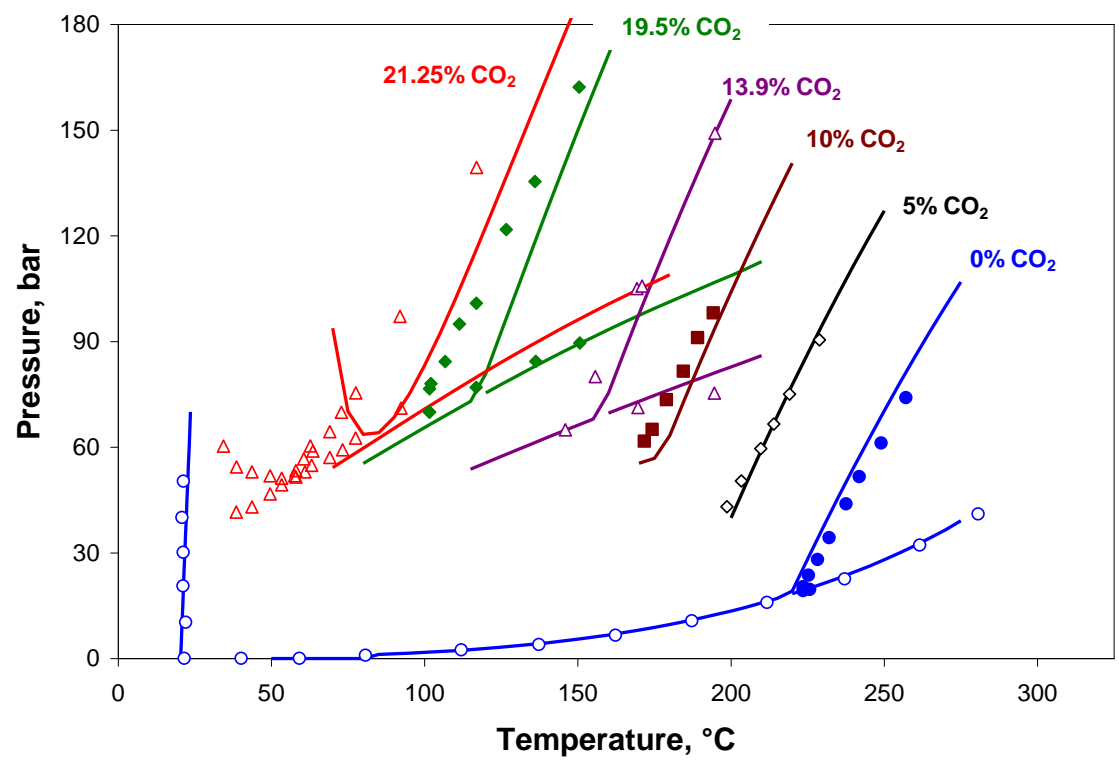

Figure 3 Cloud-point curves and vapor-liquid equilibrium of the ternary system polystyrene (PS)-cyclohexane-carbon dioxide (PS: $\mathrm{M}_{\mathrm{w}}=101.4 \mathrm{~kg} / \mathrm{mol}, \mathrm{M}_{\mathrm{w}} / \mathrm{M}_{\mathrm{n}}=1.09 ; \mathrm{w}_{\mathrm{PS}}=0.1$ at $0 \%$ $\mathrm{CO}_{2}$ ). Comparison of experimental data $[34,35]$ to PC-SAFT correlation results. The polymer is modeled as monodisperse (PS-cyclohexane, $k i j=0.0075$; $\mathrm{PS}-\mathrm{CO}_{2}, k i j=0.195$; cyclohexane$\left.\mathrm{CO}_{2}: k i j=0.13\right)($ adapted from [33]). 


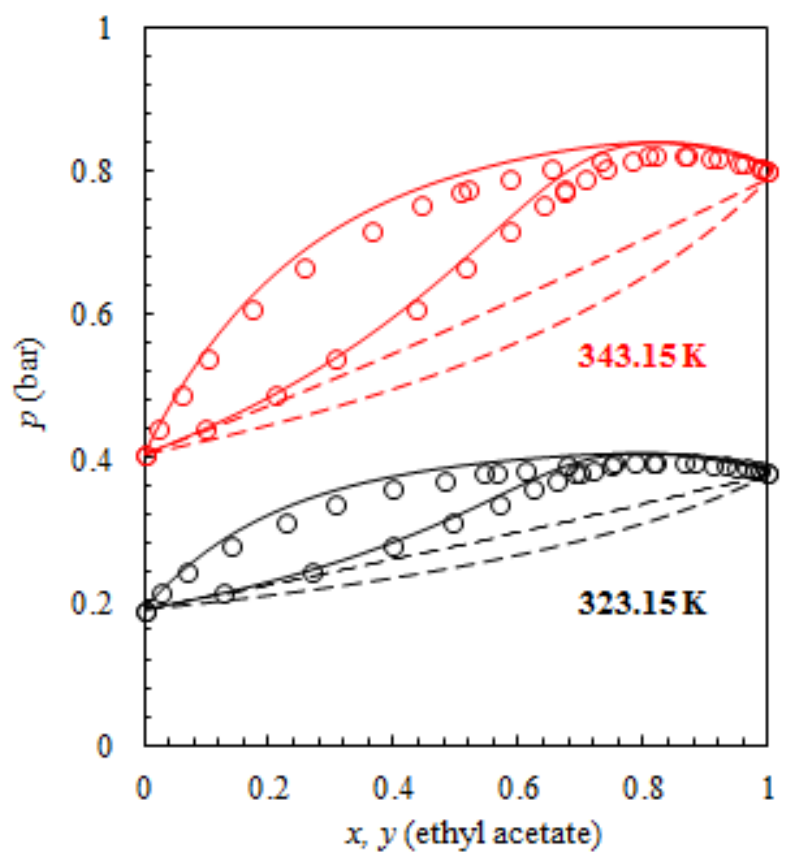

Figure 4 Vapor-liquid equilibria of the ethyl acetate + n-heptane system. Dashed line represents nonpolar PC-SAFT, and the solid line represents polar PC-SAFT. Binary interaction parameters set to zero. Symbols are experimental data from Shealy and Sandler [38] (adapted from [37]). 


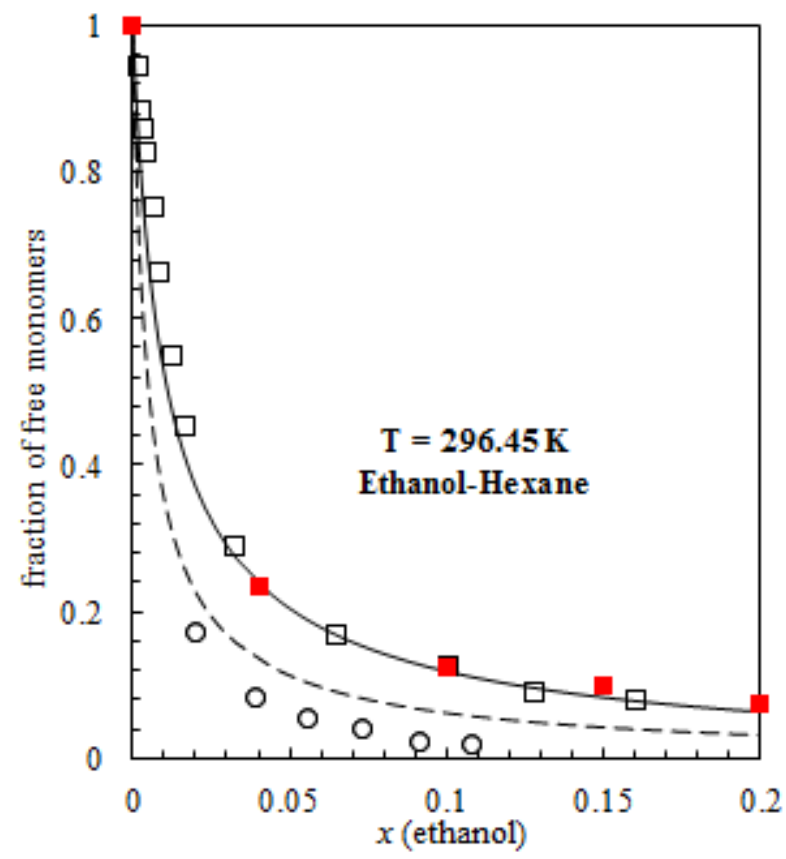

Figure 5 Free monomer fractions of the binary systems ethanol $+\mathrm{n}$-hexane as a function of composition at $296.45 \mathrm{~K}$. (o, 口): experimental data [40, 41]; (匹): MD simulations by this work; solid line (-): Polar PC-SAFT predictions; dashed line (- $)$ : PC-SAFT predictions. 


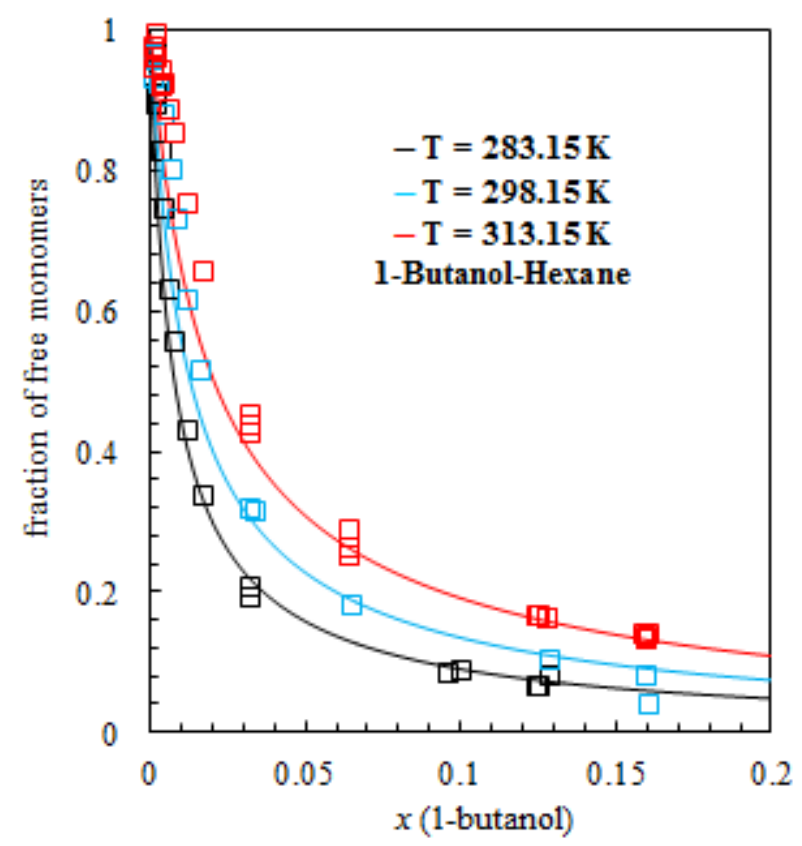

Figure 6 Free monomer fractions of the binary systems 1-butanol + n-hexane as a function of temperature and composition. ( $\square$ ): experimental data [40]; solid line (-): Polar PC-SAFT predictions. 


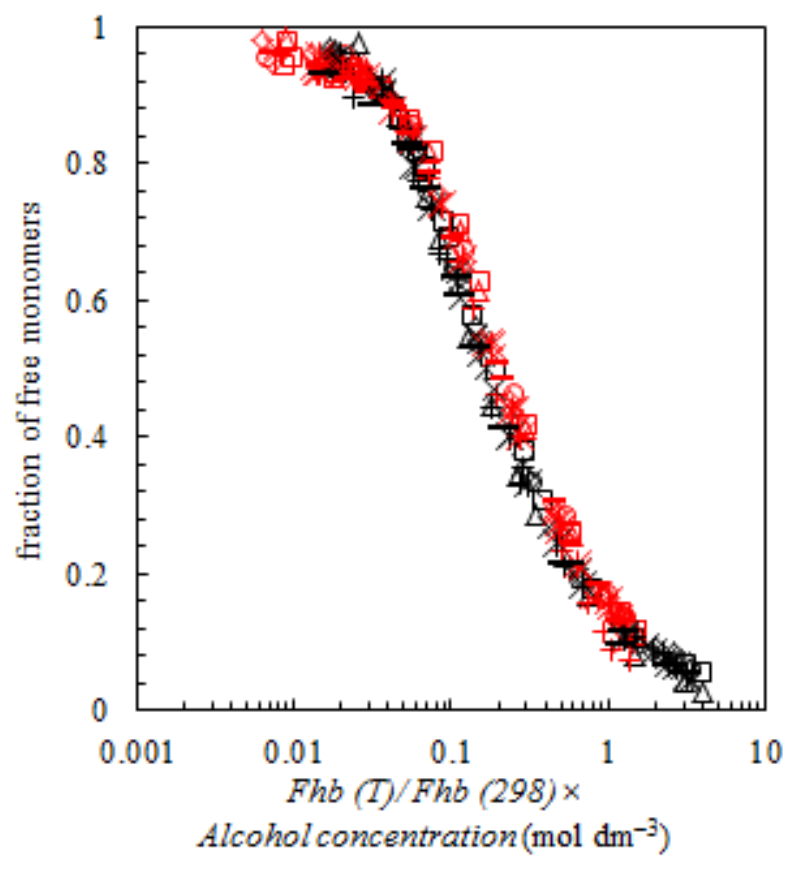

Figure 7 Experimental free monomer fractions of the binary systems alcohol + alkane at 283.15 (black) and $303.15 \mathrm{~K}$ (red) as a function of the alcohol concentration multiplied by the ratio of the Mayer f-function. FT-IR data by Asprion et al. [40]; $(\Delta, \Delta)$ : ethanol + cyclohexane; $(\square, \square)$ : 1 butanol + cyclohexane; $(+,+)$ : methanol + cyclohexane; $(0,0)$ : 1-propanol + n-hexane; $(\diamond, \diamond): 1$ butanol + n-hexane; $(-,-)$ : 1-pentanol + n-hexane; $(\times, \times)$ : 1-hexanol + n-hexane. 


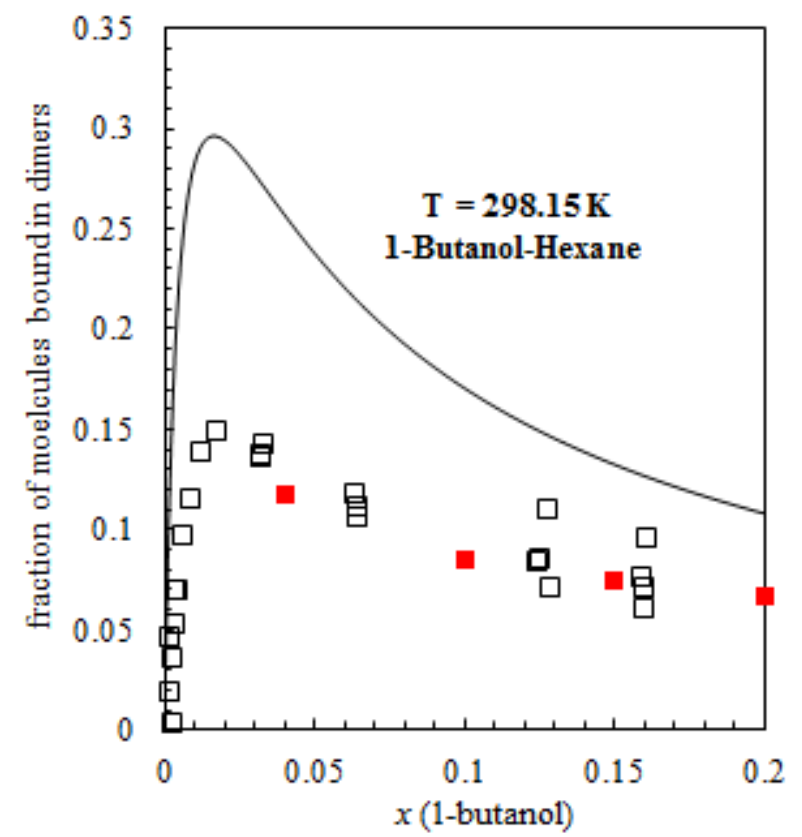

Figure 8 Fraction of molecules bound in dimers in the binary systems 1-butanol + n-hexane as a function of composition. ( $\square$ ): experimental data at 298.15 K [40]; ( $\square)$ : MD simulations by this work at 303.15; solid line (-): Polar PC-SAFT predictions. 


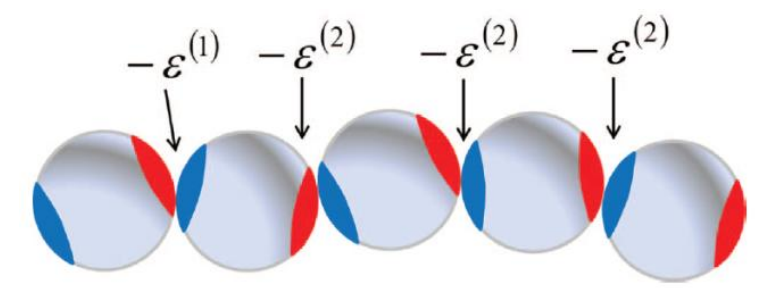

Figure 9 A scheme of a chain of associated beads, where the energy of the first bond $\varepsilon^{(1)}$ is different than other bonds energies $\varepsilon^{(2)}$. 


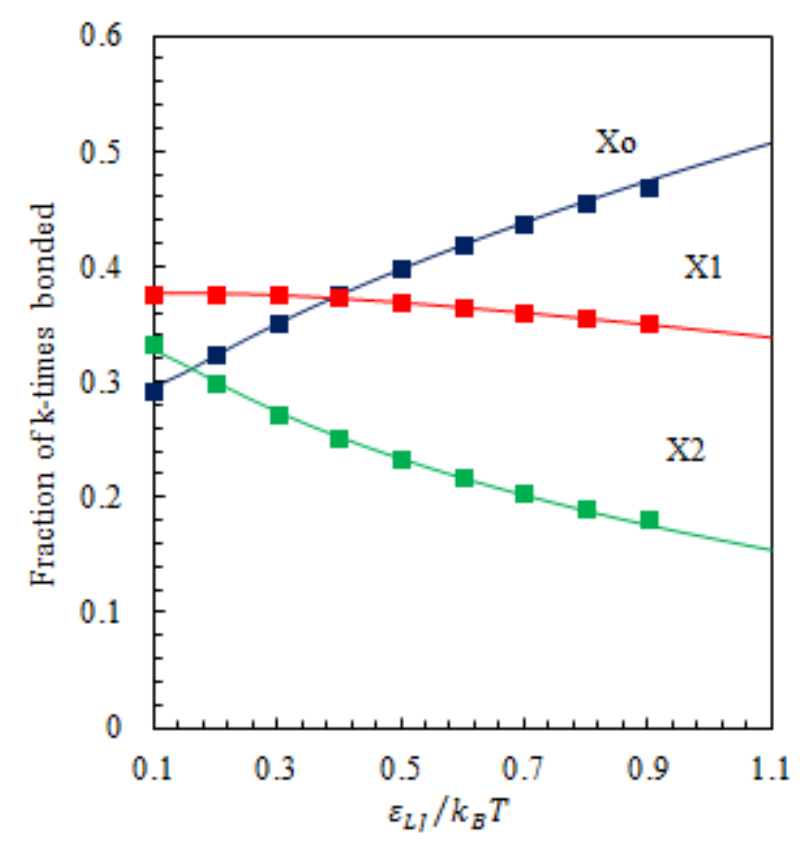

Figure 10 Fractions of k-times bonded beads versus change in reduced temperature $\left(T^{*-1}=\right.$ $\left.\varepsilon_{L J} / k_{B} T\right) . X k$ : fraction of beads bonded k-times (solid curves: theory, symbols: MC simulation), $\rho \sigma^{3}=0.6, \varepsilon_{A B}^{(1)} / k_{B} T=5.0, \varepsilon_{A B}^{(2)} / k_{B} T=6.0$. 

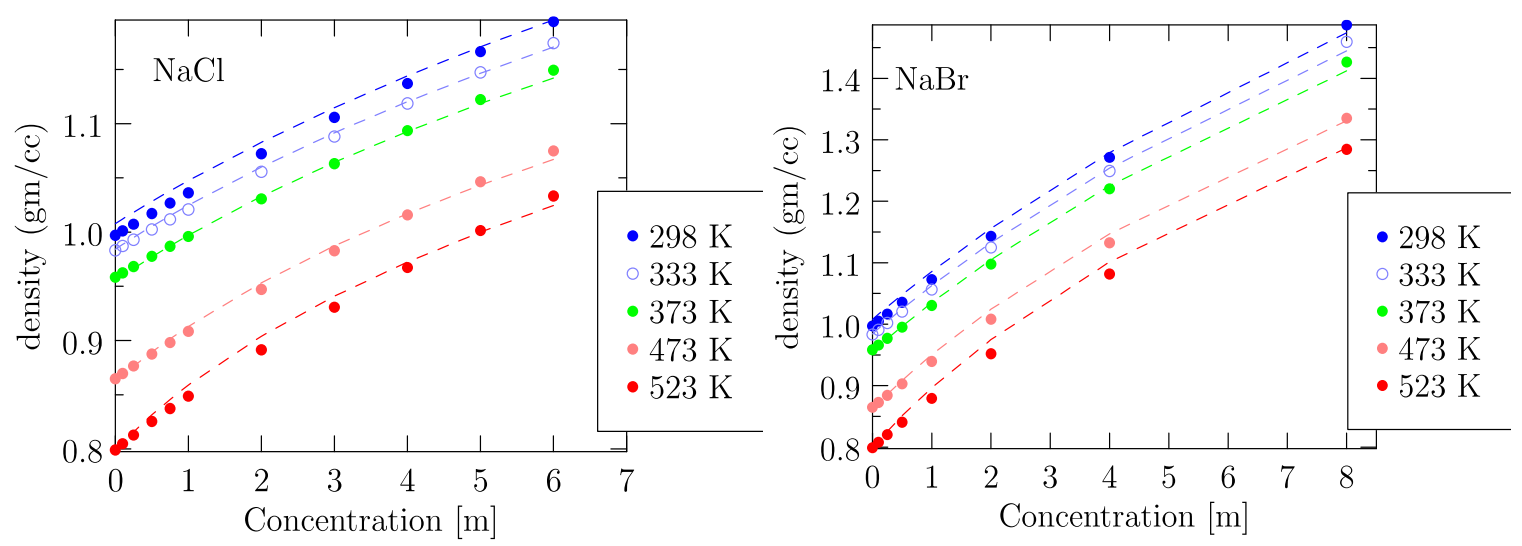

Figure 11 Density prediction at high temperature for aqueous $\mathrm{NaCl}$ (left) and $\mathrm{NaBr}$ solutions. 


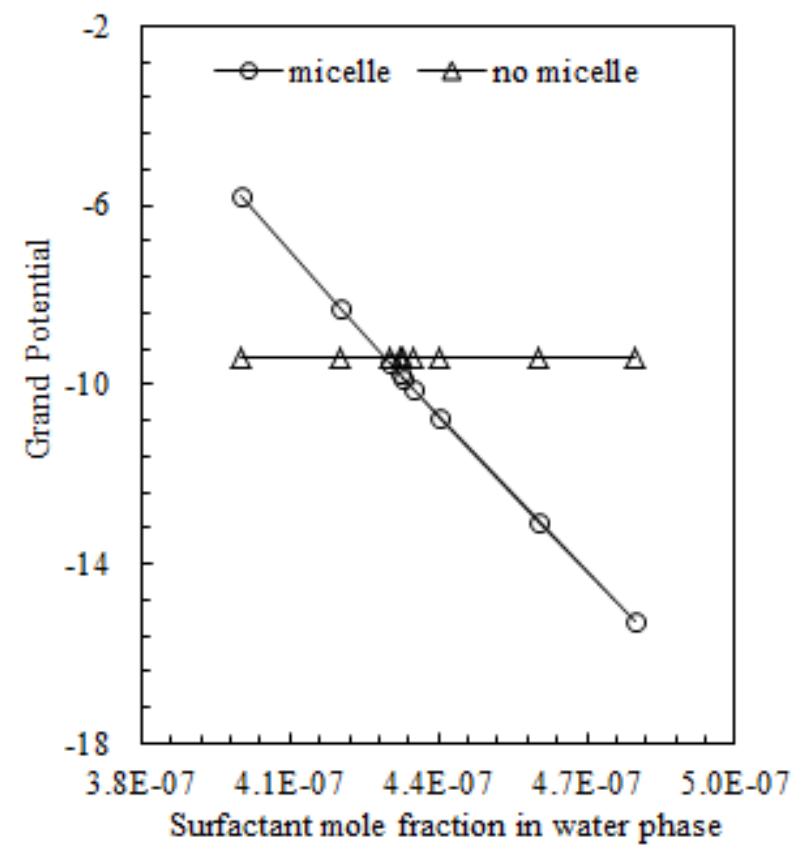

Figure 12 Grand Potential Comparison for $\mathrm{H}_{5} \mathrm{~T}_{4} / \mathrm{C}_{8} \mathrm{E}_{5}$ surfactants. 


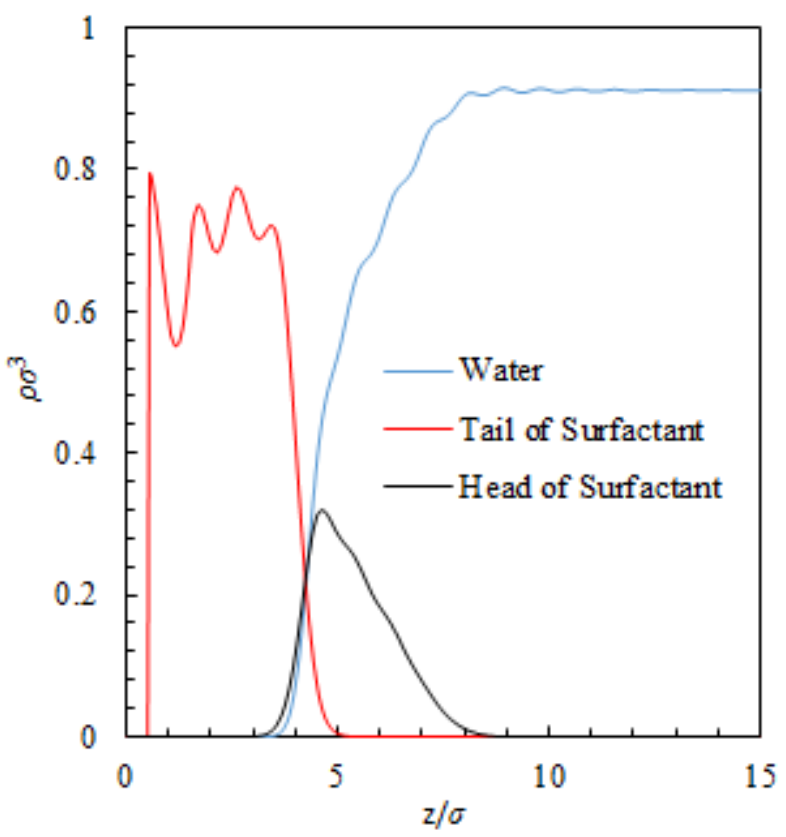

Figure 13 Structure of micelle formed by $\mathrm{H}_{5} \mathrm{~T}_{4} / \mathrm{C}_{8} \mathrm{E}_{5}$ surfactants. 
Table 1 Pure component parameters used in this work.

\begin{tabular}{|c|c|c|c|c|c|c|c|c|c|}
\hline Component & $m_{i}$ & $\begin{array}{c}\sigma_{i} \\
(\AA)\end{array}$ & $\begin{array}{c}\varepsilon_{i} / k \\
(\mathrm{~K})\end{array}$ & $\begin{array}{c}\varepsilon^{A_{i} B_{i}} / k \\
(\mathrm{~K})\end{array}$ & $\kappa^{A_{i} B_{i}}$ & $\begin{array}{c}\mu_{i} \\
(\mathrm{D})\end{array}$ & $m_{i} x_{p_{i}}$ & $\begin{array}{c}\text { AAD\% } \% \\
p^{\text {sat }}\end{array}$ & $\begin{array}{c}\text { AAD\% } \% \\
\rho^{\text {sat }}\end{array}$ \\
\hline ethanol & 2.000 & 3.428 & 230 & 2515.569 & 0.0179 & 1.70 & 0.50 & 0.93 & 1.57 \\
\hline 1-butanol & 3.000 & 3.481 & 237 & 2535.788 & 0.0125 & 1.70 & 0.50 & 3.48 & 0.25 \\
\hline n-hexane & 3.058 & 3.798 & 236.77 & & & & & 0.31 & 0.76 \\
\hline
\end{tabular}



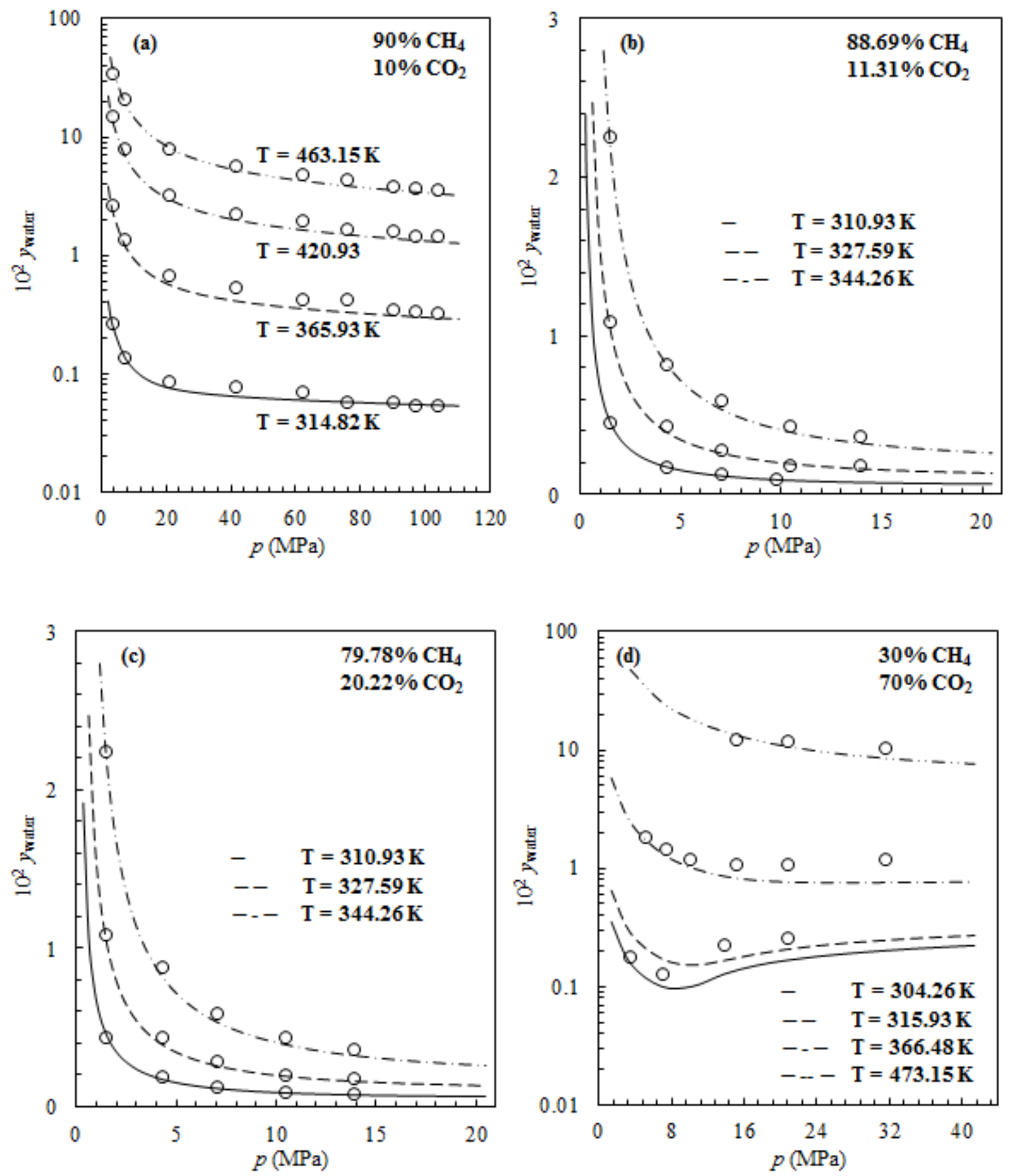

Figure 1 Water content of methane + carbon dioxide mixture as a function of temperature and pressure. (o): experimental data; (-): PC-SAFT predictions. (a): experimental data by Fouad et al. [26]; (b): experimental data by Sharma [27] for gaseous mixture of $88.69 \mathrm{~mol} . \% \mathrm{CH}_{4}$ and 11.31 mol. \% $\mathrm{CO}_{2}$; (c): experimental data by Sharma [27] for gaseous mixture of $79.78 \mathrm{~mol}$ \% $\mathrm{CH}_{4}$ and 20.22 mol. \% $\mathrm{CO}_{2}$; (d): experimental data by Fouad et al. (adapted from [26]). 

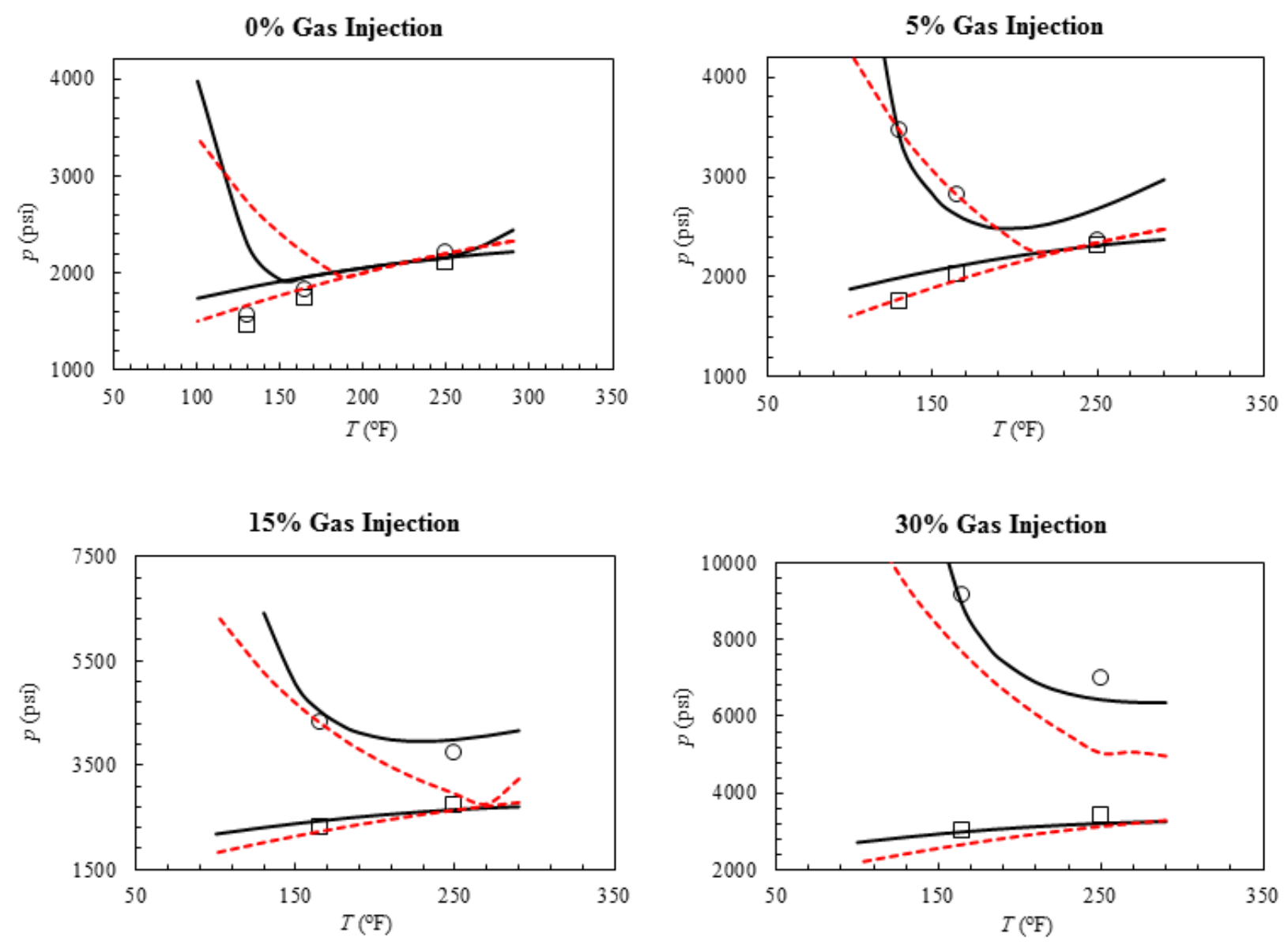

Figure 2 PC-SAFT and CPA characterized oil prediction for crude A after estimating the parameters for 5 mol. \% of gas injection data. (o): experimental AOP; ( $\square$ ): experimental bubble pressure [32]. Solid black curve (-): PC-SAFT; dashed red curve (- ): CPA. Injected gas composition (mol. \%): $\mathrm{N}_{2}-0.4 \%, \mathrm{CO}_{2}-3.9 \%, \mathrm{C}_{1}-71.4 \%, \mathrm{C}_{2}-12 \%, \mathrm{C}_{3}-7.2 \%$, heavy gas-5.1\% (adapted from [31]). 


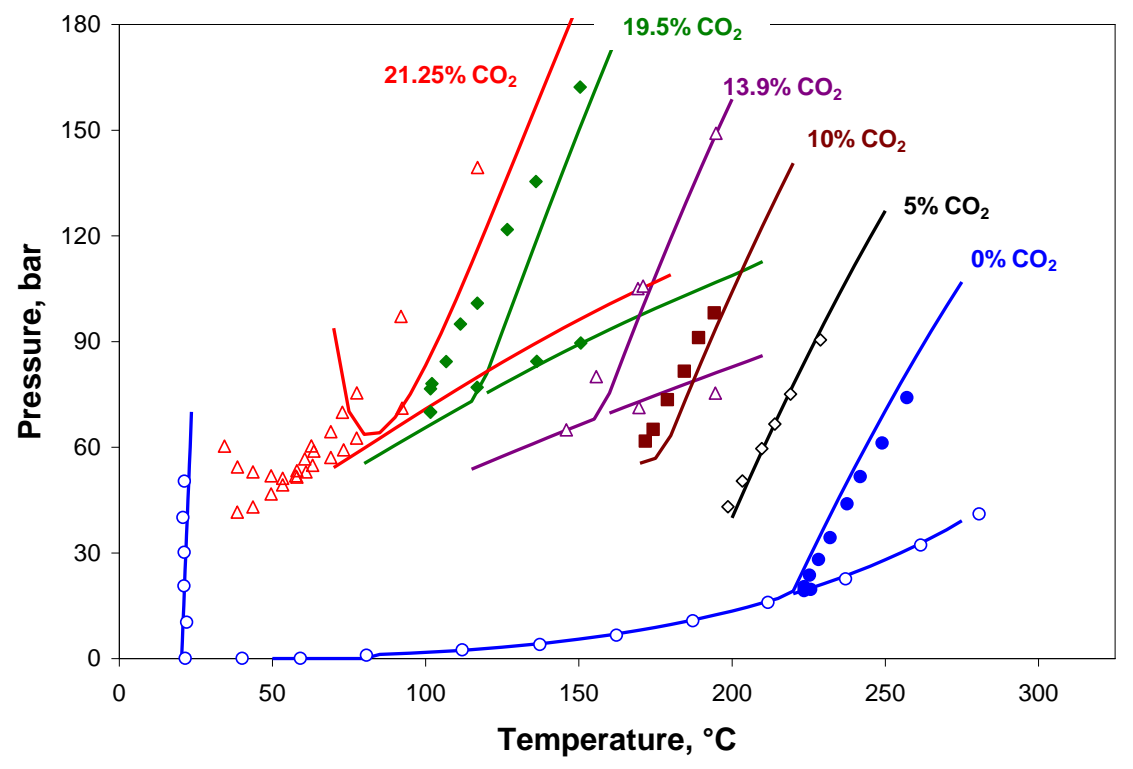

Figure 3 Cloud-point curves and vapor-liquid equilibrium of the ternary system polystyrene (PS)-cyclohexane-carbon dioxide (PS: $\mathrm{M}_{\mathrm{w}}=101.4 \mathrm{~kg} / \mathrm{mol}, \mathrm{M}_{\mathrm{w}} / \mathrm{M}_{\mathrm{n}}=1.09 ; \mathrm{w}_{\mathrm{PS}}=0.1$ at $0 \%$ $\mathrm{CO}_{2}$ ). Comparison of experimental data $[34,35]$ to PC-SAFT correlation results. The polymer is modeled as monodisperse (PS-cyclohexane, $k i j=0.0075$; $\mathrm{PS}-\mathrm{CO}_{2}, k i j=0.195$; cyclohexane$\left.\mathrm{CO}_{2}: k i j=0.13\right)($ adapted from [33]). 


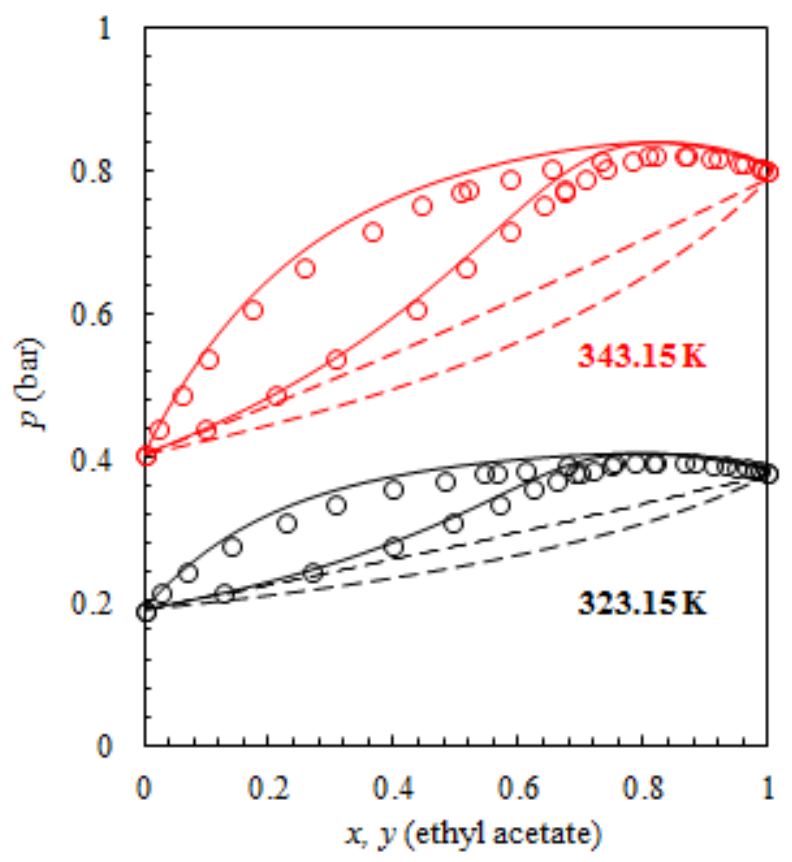

Figure 4 Vapor-liquid equilibria of the ethyl acetate + n-heptane system. Dashed line represents nonpolar PC-SAFT, and the solid line represents polar PC-SAFT. Binary interaction parameters set to zero. Symbols are experimental data from Shealy and Sandler [38] (adapted from [37]). 


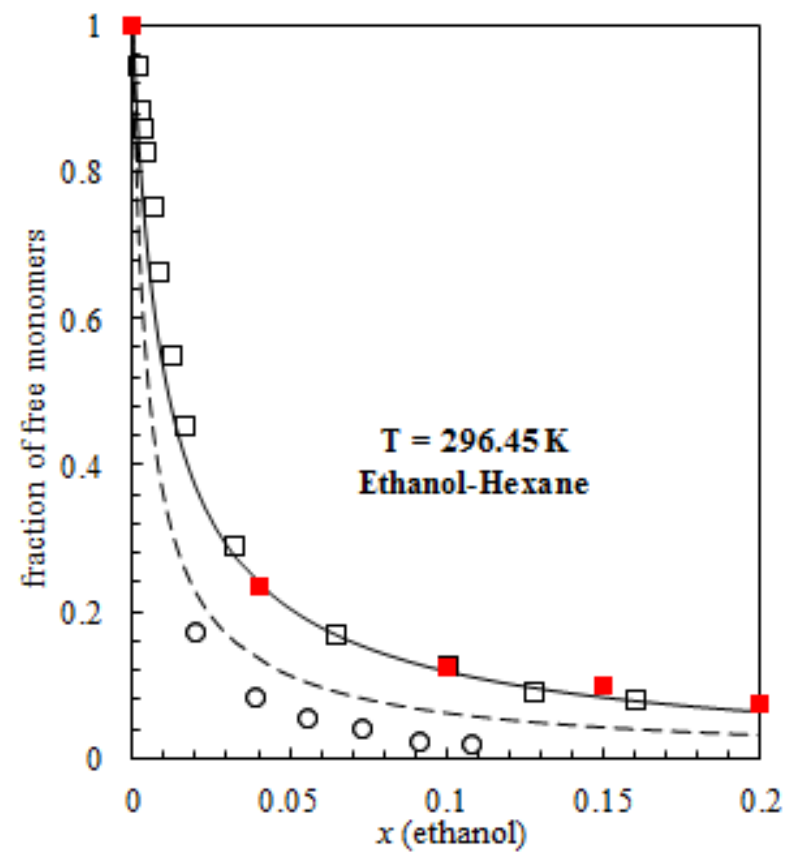

Figure 5 Free monomer fractions of the binary systems ethanol $+\mathrm{n}$-hexane as a function of composition at $296.45 \mathrm{~K}$. (o, 口): experimental data [40, 41]; (匹): MD simulations by this work; solid line (-): Polar PC-SAFT predictions; dashed line (- $)$ : PC-SAFT predictions. 


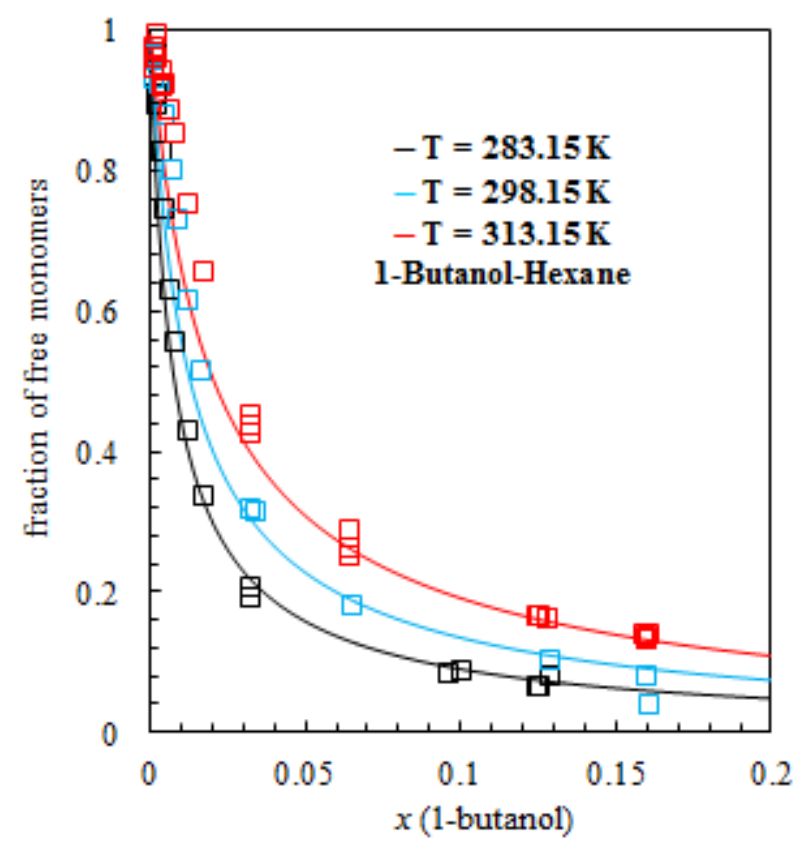

Figure 6 Free monomer fractions of the binary systems 1-butanol + n-hexane as a function of temperature and composition. ( $\square$ ): experimental data [40]; solid line (-): Polar PC-SAFT predictions. 


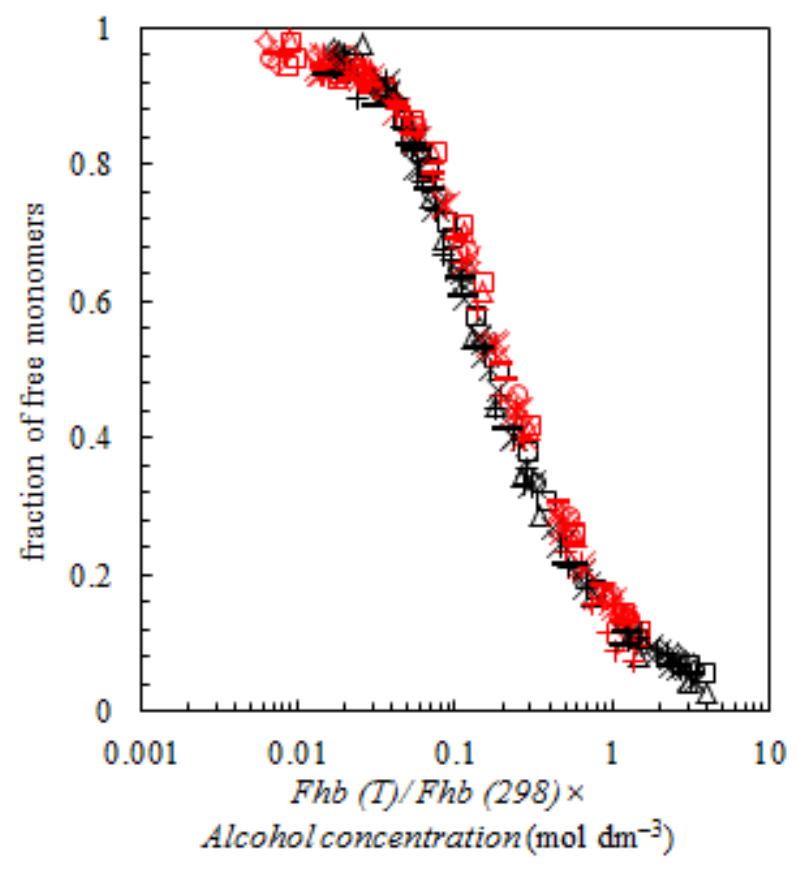

Figure 7 Experimental free monomer fractions of the binary systems alcohol + alkane at 283.15 (black) and $303.15 \mathrm{~K}$ (red) as a function of the alcohol concentration multiplied by the ratio of the Mayer f-function. FT-IR data by Asprion et al. [40]; $(\Delta, \Delta)$ : ethanol + cyclohexane; $(\square, \square)$ : 1 butanol + cyclohexane; $(+,+)$ : methanol + cyclohexane; $(0,0)$ : 1-propanol + n-hexane; $(\diamond, \diamond): 1$ butanol + n-hexane; $(-,-)$ : 1-pentanol + n-hexane; $(\times, \times)$ : 1-hexanol + n-hexane. 


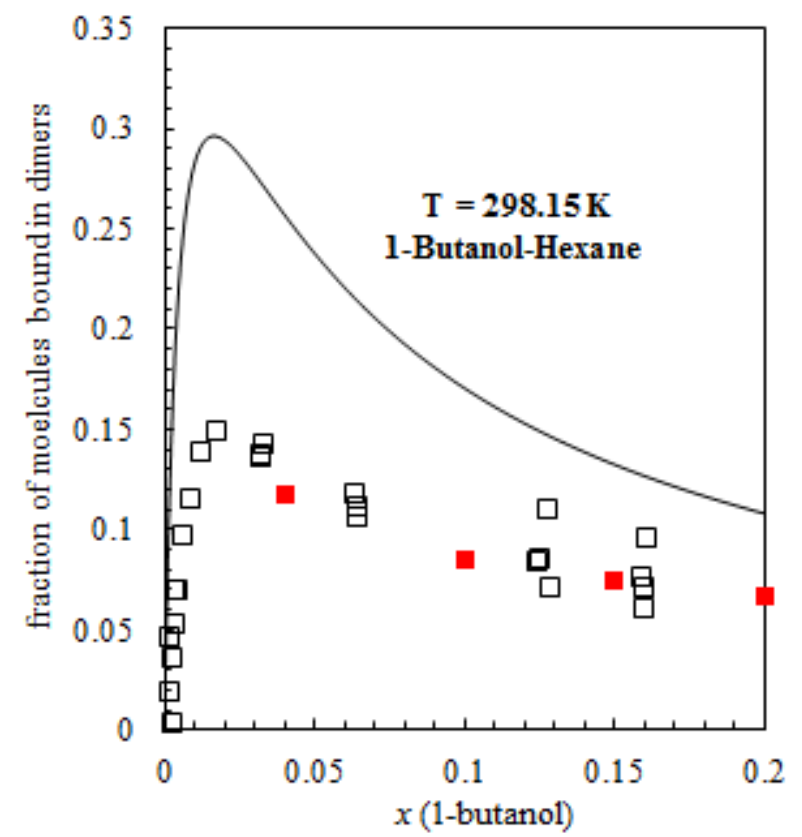

Figure 8 Fraction of molecules bound in dimers in the binary systems 1-butanol + n-hexane as a function of composition. ( $\square$ ): experimental data at 298.15 K [40]; ( $\square)$ : MD simulations by this work at 303.15; solid line (-): Polar PC-SAFT predictions. 


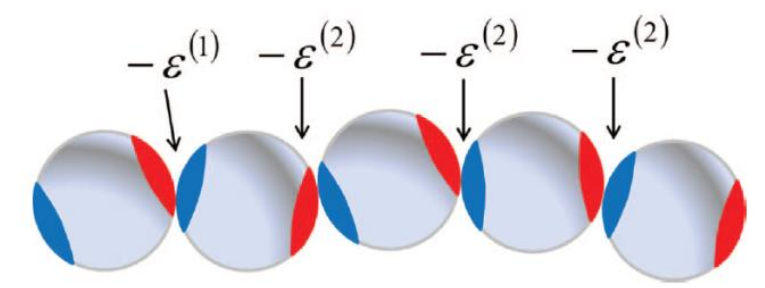

Figure 9 A scheme of a chain of associated beads, where the energy of the first bond $\varepsilon^{(1)}$ is different than other bonds energies $\varepsilon^{(2)}$. 


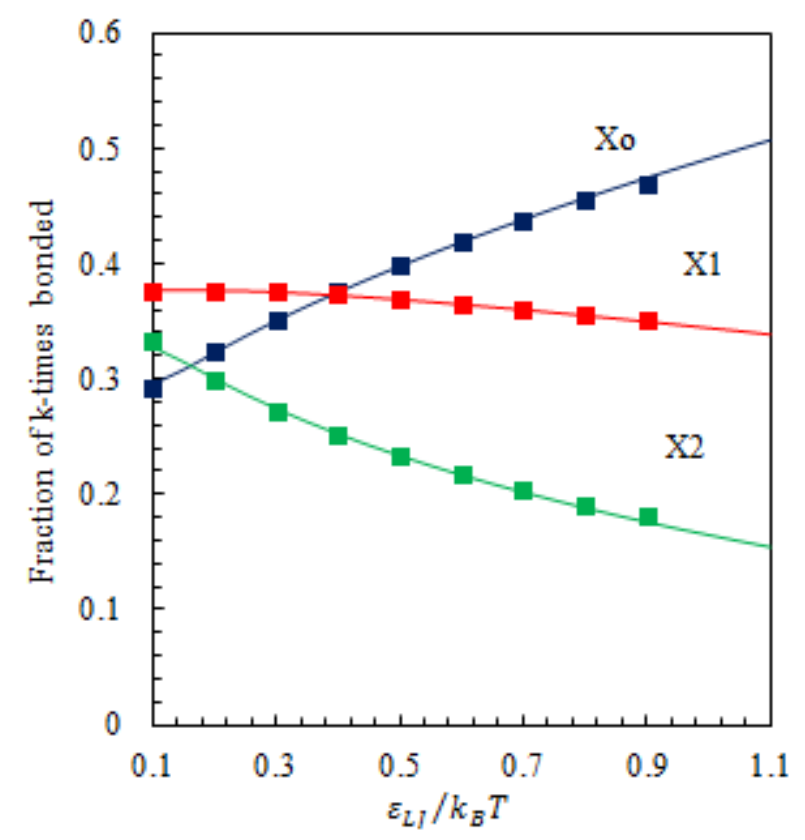

Figure 10 Fractions of k-times bonded beads versus change in reduced temperature $\left(T^{*-1}=\right.$ $\left.\varepsilon_{L J} / k_{B} T\right) . X k$ : fraction of beads bonded k-times (solid curves: theory, symbols: MC simulation), $\rho \sigma^{3}=0.6, \varepsilon_{A B}^{(1)} / k_{B} T=5.0, \varepsilon_{A B}^{(2)} / k_{B} T=6.0$. 

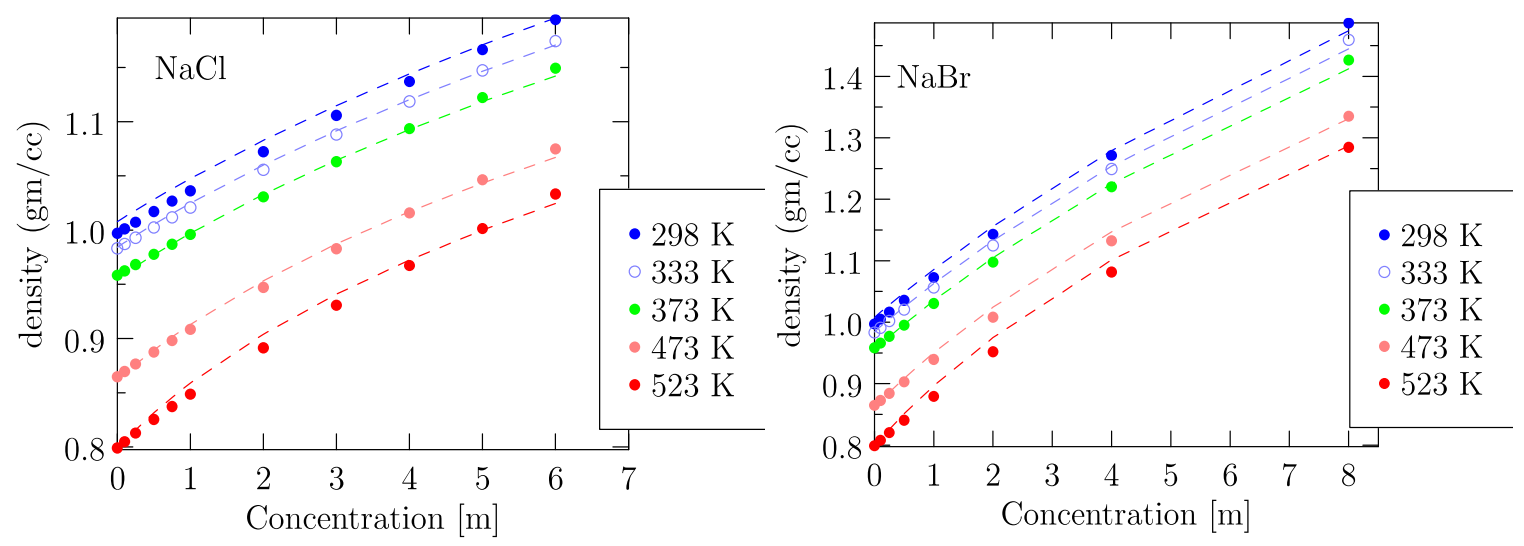

Figure 11 Density prediction at high temperature for aqueous $\mathrm{NaCl}$ (left) and $\mathrm{NaBr}$ solutions. 


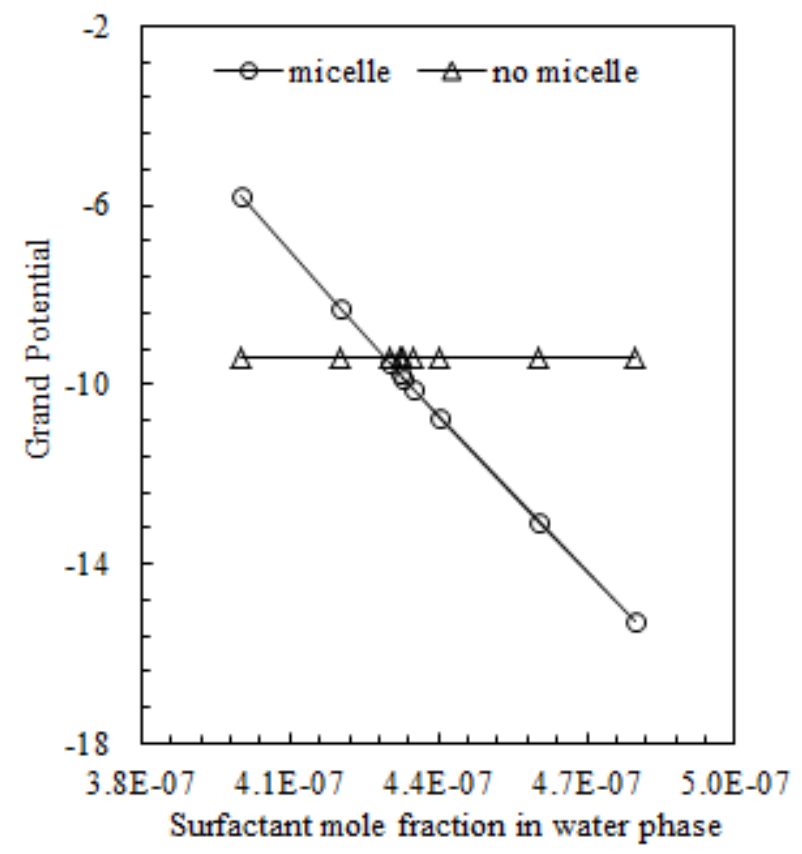

Figure 12 Grand Potential Comparison for $\mathrm{H}_{5} \mathrm{~T}_{4} / \mathrm{C}_{8} \mathrm{E}_{5}$ surfactants. 


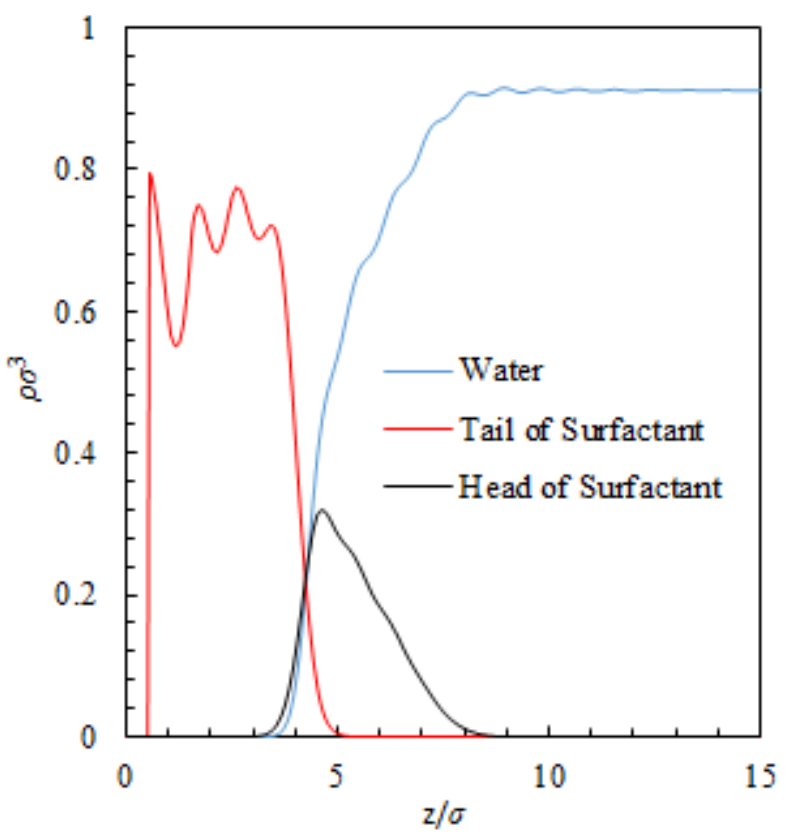

Figure 13 Structure of micelle formed by $\mathrm{H}_{5} \mathrm{~T}_{4} / \mathrm{C}_{8} \mathrm{E}_{5}$ surfactants. 